OPEN ACCESS

Edited by:

Mark Stecker

Self-Employed, Fresno, CA,

United States

Reviewed by:

Shinichi Someya,

University of Florida, United States

Angel Ballesteros,

National Institute of Neurological

Disorders and Stroke (NINDS),

United States

*Correspondence:

Dwayne D. Simmons

Dwayne_Simmons@baylor.edu

Received: 29 July 2021 Accepted: 22 October 2021 Published: 15 November 2021

Citation: Climer LK, Hornak AJ, Murtha K, Yang Y, Cox AM, Simpson PL, Le $A$ and Simmons DD (2021) Deletion of Oncomodulin Gives Rise to Early Progressive Cochlear Dysfunction in C57 and CBA Mice.

Front. Aging Neurosci. 13:749729. doi: 10.3389/fnagi.2021.749729

\section{Deletion of Oncomodulin Gives Rise to Early Progressive Cochlear Dysfunction in C57 and CBA Mice}

\author{
Leslie K. Climer', Aubrey J. Hornak ${ }^{1}$, Kaitlin Murtha ${ }^{1}$, Yang Yang ${ }^{1}$, Andrew M. Cox ${ }^{1}$, \\ Preston L. Simpson ${ }^{1}$, Andy Le ${ }^{1}$ and Dwayne D. Simmons ${ }^{1,2 *}$
}

${ }^{1}$ Department of Biology, Baylor University, Waco, TX, United States, ${ }^{2}$ Department of Psychology and Neuroscience, Baylor University, Waco, TX, United States

$\mathrm{Ca}^{2+}$ signaling is a major contributor to sensory hair cell function in the cochlea. Oncomodulin (OCM) is a $\mathrm{Ca}^{2+}$ binding protein (CaBP) preferentially expressed in outer hair cells (OHCs) of the cochlea and few other specialized cell types. Here, we expand on our previous reports and show that OCM delays hearing loss in mice of two different genetic backgrounds: CBA/CaJ and C57BI/6J. In both backgrounds, genetic disruption of $\mathrm{Ocm}$ leads to early progressive hearing loss as measured by auditory brainstem response (ABR) and distortion product otoacoustic emission (DPOAE). In both strains, loss of Ocm reduced hearing across lifetime (hearing span) by more than $50 \%$ relative to wild type (WT). Even though the two WT strains have very different hearing spans, OCM plays a considerable and similar role within their genetic environment to regulate hearing function. The accelerated age-related hearing loss (ARHL) of the Ocm KO illustrates the importance of $\mathrm{Ca}^{2+}$ signaling in maintaining hearing health. Manipulation of OCM and $\mathrm{Ca}^{2+}$ signaling may reveal important clues to the systems of function/dysfunction that lead to $A R H L$.

Keywords: oncomodulin, efferent, hearing loss, $\mathrm{Ca}^{2+}$ buffer, knockout mice, hair cells, prestin

\section{INTRODUCTION}

Approximately 15\% of American adults between the ages of 20 and 69 have high frequency hearing loss due to exposure to loud sounds, and 50\% of Americans over 75 years old are affected by agerelated hearing loss (ARHL), also known as presbycusis. ${ }^{1}$ ARHL is a progressive loss of hearing sensitivity, impaired sound localization and decreased ability to understand speech, especially in noisy environments (Bielefeld et al., 2010; Wang and Puel, 2020). Although progress has been made in defining some of the genetic and cellular functions that are disrupted by ARHL, little is known about its underlying causes. Sensory ARHL involves loss or degeneration of sensory hair cells and their neural connections in the cochlea (Lauer et al., 2012; Altschuler et al., 2015; Jeng et al., 2020a,b; Kobrina et al., 2020). Inner hair cells (IHCs) contact the majority of neural connections and transmit sound-induced impulses to the brain. Outer hair cells (OHCs) amplify and enhance IHC responses to sound. Recent studies in human temporal bones suggest that ARHL is largely affected by damage to inner ear sensory cells, particularly OHCs (Wu et al., 2020). During aging, as well as intense or prolonged noise exposure, damage to OHCs begins in basal, high frequency

\footnotetext{
${ }^{1}$ https://www.nidcd.nih.gov/health/statistics
} 
regions (Cruickshanks et al., 1998; Hu et al., 2006; Nelson and Hinojosa, 2006; Chen et al., 2009; Frisina, 2009; Wu et al., 2020). The loss of OHCs leads to elevated hearing thresholds and loss of cochlear frequency tuning. Comparisons of behavioral and physiological hearing depreciation in mice indicate that behavioral repercussions of ARHL occur earlier than the more dramatic physical changes of cochlear damage (Kobrina et al., 2020). Therefore, the molecular details of OHC dysfunction and synaptic loss are essential missing pieces in understanding the progression of ARHL in humans and mice.

Hair cell mechanotransduction, synaptic transmission and response to acoustic overstimulation depend on $\mathrm{Ca}^{2+}$ regulation (Lenzi and Roberts, 1994; Jaramillo, 1995; Fridberger et al., 1998). OHCs regulate $\mathrm{Ca}^{2+}$ through an array of channels, transporters and mobile $\mathrm{Ca}^{2+}$ buffers (Hackney et al., 2005; Fettiplace and Nam, 2019). During development, OHCs show a dramatic shift in the expression profile of the mobile $\mathrm{Ca}^{2+}$ buffer family, EFhand $\mathrm{Ca}^{2+}$ binding proteins (CaBPs). While certain CaBPs such as alpha-parvalbumin $(\alpha \mathrm{PV})$ are down regulated, oncomodulin (OCM), a member of the parvalbumin family, is upregulated in mouse OHCs soon after birth (P2-P3), supplanting other CaBPs as at the dominant CaBP (Henzl et al., 1997; Thalmann et al., 1997; Sakaguchi et al., 1998; Yang et al., 2004; Simmons et al., 2010; Climer et al., 2019). OCM is the only known mobile $\mathrm{Ca}^{2+}$ buffer that when deleted gives rise to a progressive hearing loss phenotype (Tong et al., 2016). In rodent OHCs, OCM expression is estimated to be in the millimolar range $(2-4 \mathrm{mM})$, which is similar only to $\alpha \mathrm{PV}$ expression found in fast twitch muscle (Yang et al., 2004; Hackney et al., 2005). The accelerated ARHL seen in $\mathrm{Ocm}$ KO mice strongly suggests that OCM is essential to the maintenance of hearing function in adult $\mathrm{C} 57 \mathrm{Bl} / 6 \mathrm{~J}$ mice (Tong et al., 2016). Whether OCM has a role in ARHL is unknown.

Two mouse strains, the CBA/CaJ (CBA) mouse and C57Bl/6J (C57) mouse, are frequently used as models for late- and earlyonset ARHL, respectively (Henry and Lepkowski, 1978; Henry and Chole, 1980; Greenwood, 1990; Li and Hultcrantz, 1994; Spongr et al., 1997; Jimenez et al., 1999; Frisina et al., 2011). The CBA mouse retains most of its hearing sensitivity up to 18 mo of age (in a 30-mo average lifespan), after which hearing declines progressively beginning in the high frequencies ( $\mathrm{Li}$ and Borg, 1991; Li and Hultcrantz, 1994; Spongr et al., 1997). CBA mice do not show significant hair cell loss until around 18 mo with approximately $50 \%$ of OHCs remaining in the apex and base by 26 mo (Spongr et al., 1997). In contrast, the C57 mouse has been used as a model for accelerated (early-onset) ARHL. The C57 mouse demonstrates rapid onset and progression of hearing loss compared to the CBA mouse (Li and Borg, 1991). The underlying pathology is progressive loss of cochlear sensory cells mediated by intrinsic apoptosis especially in basal hair cells after 6 mo with complete loss of basal OHCs by $12 \mathrm{mo}$ (Li and Hultcrantz, 1994; Spongr et al., 1997; Francis et al., 2003; Someya et al., 2009). Recently, Jeng et al. (2020a,b, 2021) performed extensive analyses of the biophysical properties of aging inner and $\mathrm{OHCs}$ and their synapses in four genetic mouse strains with early or late ARHL onset. They concluded that alterations in the mechanoelectrical transducer (MET) in the stereocilia bundle and $\mathrm{OHC}$ efferent synapses possibly contribute to the progression of ARHL, and that apoptosis of sensory cells may not be a contributing factor.

Given the unique expression profile of OCM in the cochlea, and the role that $\mathrm{Ca}^{2+}$ regulation may play in hearing function, we used the $\mathrm{Ocm} \mathrm{KO}$ mouse model to explore the link between OCM and ARHL. The C57 strain is known to potentiate hearing loss of other $\mathrm{Ca}^{2+}$-regulatory gene mutations (Fettiplace and Nam, 2019). Since C57 mice have an accelerated ARHL phenotype and the $\mathrm{Ocm} \mathrm{KO}$ appeared to accelerate this phenotype even further, we hypothesized that the absence of OCM in mice on the $\mathrm{CBA} / \mathrm{CaJ}$ genetic background will also induce an early progressive hearing loss phenotype but with a different time course. In this study, we compare age-related hearing decline in wild type (WT) and KO mice of both backgrounds. In both mouse strains, the $\mathrm{Ocm} \mathrm{KO}$ allele resulted in an early progressive hearing loss that was at least $50 \%$ of the normal hearing span, and a loss of efferent terminals and OHCs. Also in both strains, aged WT mice show alterations in the localization of OCM that coincide with the loss of OHCs and efferent terminals. This study suggests that OCM may indeed play a role in ARHL associated with OHC dysfunction.

\section{MATERIALS AND METHODS}

\section{Animals}

All experiments were done in compliance with National Institutes of Health and institutional animal care guidelines and were approved by the Institutional Animal Care and Use Committee of Baylor University. $\mathrm{Ocm}^{-/-}(\mathrm{Ocm} \mathrm{KO})$ mice were generated from spontaneous germline transmission of the $\mathrm{KO}$ allele from the parental line, $A c t b^{C r e} ; \mathrm{Ocm}^{\text {flox/flox }}$ on the C57Bl/6J background (Tong et al., 2016). The original C57 Ocm KO mice were backcrossed onto the CBA/CaJ background for 10 generations. At each generation, mice were genotyped for the Cdh23-ahl locus and only animals with the CBA/CaJ Cdh23 allele were bred following procedures used by Liberman and colleagues (Liu et al., 2007). The Cdh23 WT or ahl gene region was amplified using primers that would generate a $360 \mathrm{bp}$ product. $C d h 23^{W T / a h l}$ For: 5'-GATCAAGACAAGACCAGACCTCTGTC-3'. Rev: 5' GAGCTACCAGGAACAGCTTGGGCCTG-3'. The PCR product was sequenced to confirm lack of $C d h 23^{a h l}$ (Supplementary Figures 1A,B). Confirmation of congenicity was done by whole genome scan (Jackson Laboratories, Bar Harbor, ME, United States). Our C57 Ocm KO mice were crossed with B6.129P2-Pvalbtm1Swal/J on the C57Bl/6J background by Jackson Laboratories (Bar Harbor, ME, United States). Homozygous CBA Ocm KO and C57 Ocm KO and WT littermates from each sex were used for all experiments.

\section{Antibodies}

Tissues were incubated with primary antibody overnight at $37^{\circ} \mathrm{C}$. Primary antibodies used: goat anti-OCM, (1:500, Santa Cruz sc7446, AB_2267583), rabbit anti-prestin [1:5,000, kindly provided by Robert Fettiplace (Furness et al., 2008)], goat anti-choline acetyltransferase [ChAT, 1:500, Millipore, AB144P, AB 2079751, (Misgeld et al., 2002)]. All primary antibodies were labeled with 
species appropriate Alexa Fluor (ThermoFisher) or Northern Lights (R\&D Systems) secondary antibodies for $2 \mathrm{~h}$ at $37^{\circ} \mathrm{C}$.

\section{Cochlear Function Assays}

For measurement of auditory brainstem responses (ABRs) and distortion product otoacoustic emissions (DPOAEs), adult mice were anesthetized with xylazine $(20 \mathrm{mg} / \mathrm{kg}$, i.p. $)$ and ketamine $(100 \mathrm{mg} / \mathrm{kg}$, i.p.). Acoustic stimuli were delivered using a custom acoustic assembly previously described by Maison et al. (2012). Briefly, two electrostatic earphones (EC-1, Tucker Davis Technologies) were used to generate primary tones and a Knowles miniature microphone (EK-3103) was used to record ear-canal sound pressure. Stimuli were generated digitally with 4s sampling. Ear-canal sound pressure and electrode voltage were amplified and digitally sampled at 20s for analysis of response amplitudes. Both outputs and inputs were processed with a digital I-O board (National Instruments PXI-4461). For measurement of ABRs, needle electrodes were inserted at vertex and pinna, with a ground electrode near the tail. ABR potentials were evoked with $5 \mathrm{~ms}$ tone pips $(0.5 \mathrm{~ms}$ rise-fall with a cos 2 onset, delivered at $35 / \mathrm{s})$. The response was amplified $(10,000)$, filtered ( $100 \mathrm{~Hz}-3 \mathrm{kHz})$, digitized, and averaged in a LabVIEWdriven data-acquisition system. Sound level was raised in $10 \mathrm{~dB}$ steps from $10 \mathrm{~dB}$ below threshold up to $80 \mathrm{~dB}$ sound pressure level (SPL). At each sound level, 1,024 responses were averaged (with stimulus polarity alternated), using an "artifact reject" whereby response waveforms were discarded when peak-to-peak amplitude exceeded 15V (e.g., electro-cardiogram or myogenic potentials). Threshold was defined as the lowest SPL level at which wave-I peak could be identified, usually corresponding to the level step just below that at which the peak-to-peak response amplitude rose significantly above the noise floor. For amplitude vs. level functions, the wave-I peak was identified by visual inspection at each sound level and the peak-to-peak amplitude computed. For measurement of DPOAEs at $2 \mathrm{f} 1-\mathrm{f} 2$, the primary tones were set so that the frequency ratio, (f2/f1), was 1.2 and so that $\mathrm{f} 2$ level was $10 \mathrm{~dB}$ below $\mathrm{f} 1$ level. For each $\mathrm{f} 2 / \mathrm{f} 1$ primary pair, levels were swept in $10 \mathrm{~dB}$ steps from $20 \mathrm{~dB}$ SPL to $80 \mathrm{~dB}$ SPL (for f2). At each level, both waveform and spectral averaging were used to increase the signal-to-noise ratio of the recorded ear-canal sound pressure, and the amplitude of the DPOAE at $2 \mathrm{f} 1$ - $\mathrm{f} 2$ was extracted from the averaged spectra, along with the noise floor at nearby points in the spectrum. Iso-response curves were interpolated from plots of DPOAE amplitude vs. sound level. Threshold was defined as the $\mathrm{f} 2$ level required to produce a DPOAE at $0 \mathrm{~dB}$ SPL. We used a total of 25 female and 18 male WT and 13 female and 13 male KO CBA mice, and a total of 40 female and 38 male WT and 17 female and 25 male KO C57 mice. Right ears were used for all hearing tests. For both background strains, variances between female and male mice were negligible across the ages tested.

\section{Immunocytochemistry}

For histological analysis and immunocytochemistry, anesthetized mice (Euthasol, $150 \mathrm{mg} / \mathrm{kg}$, i.p.) were perfused transcardially with $4 \%$ paraformaldehyde (PFA) in $0.1 \mathrm{M}$ phosphate buffer (PBS) followed by PBS wash. After removal, cochleae scalae were flushed with PFA then rotated in fixative overnight at $4^{\circ} \mathrm{C}$. Cochleae were decalcified in $0.1 \mathrm{M}$ EDTA for $3-5$ days at $4^{\circ} \mathrm{C}$ with rotation. Cochleae were prepared as cochlear whole mounts of approximately 6 pieces of sensory epithelium or as $100 \mu \mathrm{m}$ mid-modiolar sections embedded in gelatin-agarose solution (Simmons et al., 2010; Maison et al., 2012). Cochlear pieces were suspended in 30\% sucrose for 30-60 min at room temperature with gentle shaking, frozen at $-80^{\circ} \mathrm{C}$ for $30 \mathrm{~min}$, then thawed for $30 \mathrm{~min}$ at $37^{\circ} \mathrm{C}$. Pieces were washed 3 times in PBS, then blocked in $5 \%$ normal horse serum (NHS) for $2 \mathrm{~h}$ at room temperature. Images were acquired using the LSM800 microscope (Zeiss) using a high-resolution, oil-immersion objective (Plan-Apochromat $63 \mathrm{x}, 1.4 \mathrm{NA}$ ). Cohorts of samples were immunostained at the same time and imaged under the same optical conditions to allow direct comparisons.

\section{Hair Cell and Efferent Cluster Counts}

Low-power images of each microdissected piece of surface preparations were obtained with a $10 \mathrm{x}$ air objective (N.A. 0.3) on a LSM800 confocal microscope. Cytocochleograms were constructed from these images by tracing the cochlear spiral and superimposing hash marks using a custom ImageJ Measure Line plugin from Eaton-Peabody. ${ }^{2}$ The plugin superimposed frequency correlates on the microdissected spiral image by application of the cochlear frequency map for mice. Organ of Corti was imaged at the 8,16 , and $32 \mathrm{kHz}$ regions with the LSM800 confocal microscope using a $63 \mathrm{x}$ oil immersion objective (N.A. 1.4). At each of the desired locations, 3 adjacent microscopic fields (9-12 IHCs per field) were imaged with a 4channel z-stack spanning the height of the hair cells to capture the stereocilia, nuclei and synaptic junctions. Hair cells were only counted if they had a nucleus or hair bundle/cuticular plate present. ChAT-labeled efferent clusters of terminal swellings were counted in equally spaced distance regions using a LSM 5 confocal microscope using a 40x oil immersion objective (N.A. 1.2). In each cochlear location, we acquired two adjacent $\mathrm{z}$-stacks (each spanning $112 \mu \mathrm{m}$ of the cochlear spiral), taking care to span the entire region containing ChAT-positive terminals along the $z$-axis. Because of the difficulty counting ChAT-labeled terminals in older animals, we resorted to counts only of clusters of labeled efferent terminals. Counts were performed by a blinded observer.

\section{Oncomodulin Subcellular Distribution}

For OCM subcellular distribution, quantification was performed in ImageJ using a region of interest (ROI) from the nucleus compared with a similar ROI in the cytoplasm. After background removal, cytoplasmic OCM fluorescence intensity was divided by nuclear OCM fluorescence intensity. 2-5 cochlea from different animals were analyzed per condition.

\section{Statistical Analysis}

All statistical analysis were performed in Prism (v9.1x GraphPad Software). All data were tested for homogeneity of variance. For multiple comparisons, Brown-Forsythe and Welch ANOVA

${ }^{2}$ https://www.masseyeandear.org/research/otolaryngology/eaton-peabodylaboratories/histology-core 
test was applied when SDs are significantly different $(p<0.05)$, followed by Dunnett's multiple comparisons test. Mann-Whitney U-test was applied when normal distribution cannot be assumed. Mean values are quoted in text and figures as means \pm SEM (DPOAE and ABR measurement) and means \pm SD (missing OHC/ChAT counting and OCM distribution). $p<0.05$ was selected as the criterion for statistical significance.

\section{RESULTS}

\section{Oncomodulin Delays Progressive Hearing Loss}

We previously showed that targeted deletion of $\mathrm{Ocm}$ in $\mathrm{C} 57 \mathrm{Bl} / 6 \mathrm{j}$ mice have elevated hearing thresholds by $3-4$ mo (Tong et al., 2016). We wanted to compare the contribution of OCM to auditory function in mice with different genetic backgrounds, specifically, C57 mice with short hearing lifespans to CBA mice with longer hearing spans. We transferred the $\mathrm{Ocm} \mathrm{KO}$ allele from the original C57 mouse line to the CBA background (see section "Materials and Methods"). To test hearing function, $\mathrm{ABR}$ waveforms of $\mathrm{C} 57 \mathrm{KO}$ (Figures 1A-D) and CBA KO (Figures 1E-H) mice at early adult ages up to the ages where $\mathrm{ABR}$ potential responses were lost were compared with agematched WT C57 and CBA mice. ABR potentials at 8 and $12 / 16 \mathrm{kHz}$ frequencies are similar for WT and $\mathrm{KO}$ mice at 2 mo in both backgrounds (Figures 1A,B,E,F). Ocm KOs showed a lack of response under these same conditions as early as $4 \mathrm{mo}$ in C57 mice (Figures 1C,D) and 7 mo in CBA mice (Figures 1G,H), whereas the WT animals demonstrated robust ABR potentials at $8 \mathrm{mo}$ in both backgrounds. $\mathrm{C} 57 \mathrm{Ocm}$ KO ABR thresholds shifted $20-60 \mathrm{~dB}$ at middle and high frequencies between 1 and 3 mo of age (Tong et al., 2016). Tong et al. (2016) showed a progressive elevation of ABR thresholds in the $\mathrm{C} 57 \mathrm{KO}$. In CBA mice, we measured ABR thresholds at 3 different ages using 8,16 , and $32 \mathrm{kHz}$ using the appearance of the wave-I peak as the criteria for threshold (Figures 1I,J). WT and KO animals showed similar thresholds at $2 \mathrm{mo}$, but $\mathrm{KO}$ animals showed threshold shifts by 5 mo, particularly at higher frequencies, and were essentially deaf by 7 mo (Figure 1I). In the CBA KO, 2 - 7 mo DPOAE thresholds were significantly different from each other at $16 \mathrm{kHz}$ (Welch's ANOVA: $p=0.0003$; Dunnett's post-test: $p=0.0002$ for $2-7 \mathrm{mo}$ ). In contrast, WT animals demonstrate similar ABR thresholds at low and middle frequencies from 2 to $7 \mathrm{mo}$, and only show substantial threshold shifts in the high frequencies at $5-7$ mo and middle frequencies at a year (Figure 1J). At $16 \mathrm{kHz}$ in the CBA WT, there was no significant difference in ABR thresholds up to 7 mo (Welch's ANOVA: $p=0.104$, followed by Dunnett's test).

To confirm that germ-line transmission of the $\mathrm{Ocm} \mathrm{KO}$ in $\mathrm{C} 57 \mathrm{Bl} / 6 \mathrm{~J}$ mice did not change the original hearing loss phenotype described by Tong et al. (2016), we measured DPOAE thresholds in age-matched $\mathrm{KO}$ and WT C57 and CBA mice over the course of their hearing span (Figures 2A-F). The C57 WT control mice showed little threshold shifts between 1 and 5 mo. The C57 KO mice show a progressive elevation of DPOAE thresholds from 1 to 6 mo (Figure 2A). At $16 \mathrm{kHz}$, there were significant differences in DPOAE thresholds between $1-3$ mo and $3-6$ mo (Welch's ANOVA: $p<0.0001$; Dunnett's test: $p=0.009$ for $1-3 \mathrm{mo}, p=0.002$ for $3-6 \mathrm{mo})$. In the $\mathrm{C} 57 \mathrm{KO}$ between 3 and $6 \mathrm{mo}$, we observed sizable threshold shifts in all but the lowest frequencies $(8-32 \mathrm{kHz})$, while in the WT, sizable threshold shifts were observed between 5 and 16 mo (Figures 2A,B). The largest threshold shifts in the C57 KO were in the middle frequencies. Although C57 KO mice were at or near the limit of DPOAE threshold detection by $6 \mathrm{mo}$, the C57 WT mice had measurable DPOAEs in most frequencies at $16 \mathrm{mo}$. In the C57 WT, there were no significant differences in $16 \mathrm{kHz}$ thresholds of 1,4 , and 5 mo mice (Dunnett's test). There was a significant difference in thresholds between 1 and $16 \mathrm{mo}$ at $16 \mathrm{kHz}(p=0.009$, Dunnett's test). Since OCM is predominantly expressed in OHC of the organ of Corti at these ages, these results confirm that $\mathrm{OHC}$ defects are primarily responsible for the differences in $\mathrm{ABR}$ potentials between the $\mathrm{Ocm} \mathrm{KO}$ early onset hearing loss and the WT.

Similar to ABR threshold responses (Figure 1I), CBA KO animals showed middle- to high-frequency DPOAE threshold shifts from 2 to 5 mo (Figure 2C). The KOs also showed nearly maximum threshold shifts at all frequencies from 7 to $12 \mathrm{mo}$. At $16 \mathrm{kHz}$, DPOAE thresholds were not statistically different between 2 and 5 mo but were significantly different between 2 and 7 mo and $2-12$ mo (Welch's ANOVA: $p<0.0001$; Dunnett's post-test: $p<0.0001$ for $2-7 \mathrm{mo}, p<0.0001$ for $2-12 \mathrm{mo}$ ). In contrast, WT mice maintained healthy threshold levels at least up to 16 mo of age and produced measurable DPOAE threshold responses in most frequencies by 28 mo (Figure $2 \mathrm{D}$ ).

At $16 \mathrm{kHz}$ in CBA mice, only DPOAE thresholds comparisons between 2 and 28 mo were significantly different $(p<0.0005$, Dunnett's test). Interestingly, young adult KO mice ( $2 \mathrm{mo}$ ) show enhanced DPOAE input-output functions compared with WT animals suggesting enhanced electromotility at least for higher frequencies. We compared DPOAE input-output functions at $32 \mathrm{kHz}$ across ages (Figures 2E,F). The $\mathrm{KO}$ animals required less signal (dB SPL) to produce a DPOAE output that crosses threshold (Figure 2E). This efficiency is lost by $5 \mathrm{mo}$, and by 12 mo the KO input-output responses do not cross threshold. WT animals lose their input-output efficiency between 5 and 12 mo, requiring higher $\mathrm{dB} S \mathrm{SP}$ input to cross threshold than at younger ages (Figure 2F). Overall, the only significant difference in amplitudes in the CBA WT were between the 2 mo and 28 mo animals $(p<0.05)$.

Taken together, $\mathrm{Ocm} \mathrm{KO}$ reduces the hearing lifetime (hearing span) of mice regardless of genetic background (Figure 3). Though CBA mice are a well-established mouse model that hears throughout most its life, Ocm deletion in OHCs reduces the hearing span to less than half of the time of WT counterparts with severe hearing dysfunction in the first 1/3 of life (Figure 3, 2nd bar). Deletion of $\mathrm{Ocm}$ similarly reduces the hearing span of C57 mice, but at an earlier age. When comparing hearing thresholds between the two genetic backgrounds, loss of OCM in the CBA background recapitulates a similar hearing phenotype of the C57 background (Figure 3, middle bars). These data demonstrate that 


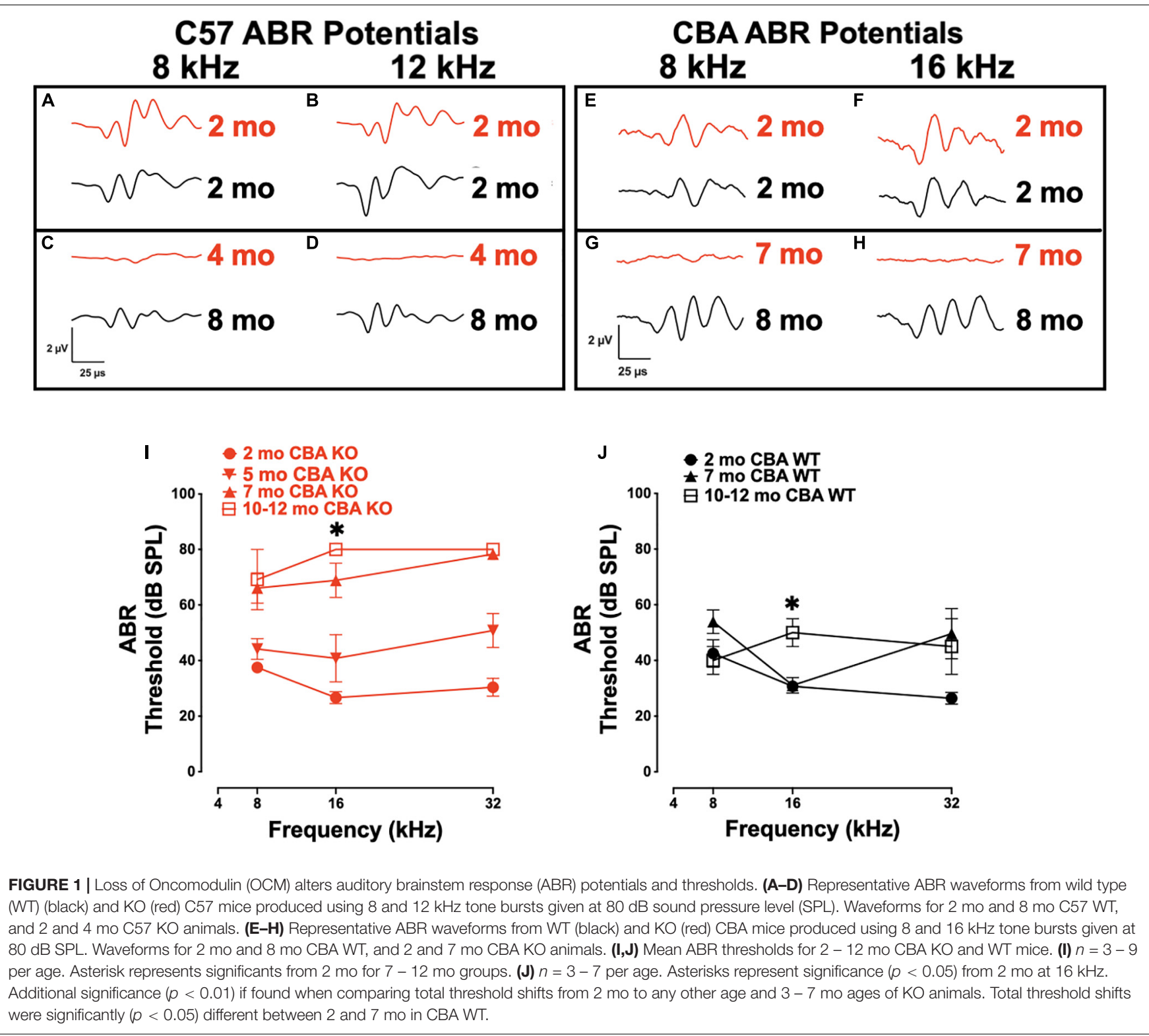

OCM is an essential contributor to hearing health by delaying progressive hearing loss as a function of age.

\section{Oncomodulin and Prestin Are Expressed in Defective Outer Hair Cells}

Since $\mathrm{Ocm}$ KO mice appear to accelerate early progressive hearing loss, we wanted to compare aging OHC phenotypes in the WT and $\mathrm{KO}$ mice across genetic backgrounds. In CBA animals, WT animals manifest substantial OHC loss after 1 year (Figures 4A-C,I). Interestingly, OCM expression is maintained even when there is substantial OHC loss. However, the subcellular localization and intensity of OCM immunostaining varies with age and frequency region across genetic background. Surface preparations were collected, stained and imaged under the same optical conditions to visualize OCM redistribution and apparent expression by age. In CBA WT mice, we find OCM immunoreactivity is more intense in the nucleus than in the cytosol of 2-month-old animals in the 8,16 , and $32 \mathrm{kHz}$ regions (Figure 4 and Supplementary Figures 1C-F, 2A,B). OCM expression becomes more cytoplasmic with age with a few cells with intensely labeled OCM in the cytosol and lateral membrane at 12 mo (Figures $4 \mathrm{~A}-\mathrm{C}, 12 \mathrm{mo}^{*}$ ). This phenomenon is more extreme in basal regions and at older ages (Supplementary Figures 2A,B). Additionally, the WT OHC damage and/or loss was more pronounced in apical regions beginning at 1 year and becoming more pronounced by 30 months (Figures 4,5 ). Since we know that WT animals have poorer hearing thresholds from 12 to $30 \mathrm{mo}$, it is possible that the intense OCM expression profile of older animals (Figure 4 and Supplementary Figures 2A,B) is a feature of dysfunctional/aging OHCs. Older animals still maintain expression of the $\mathrm{OHC}$ specific motor protein prestin, 

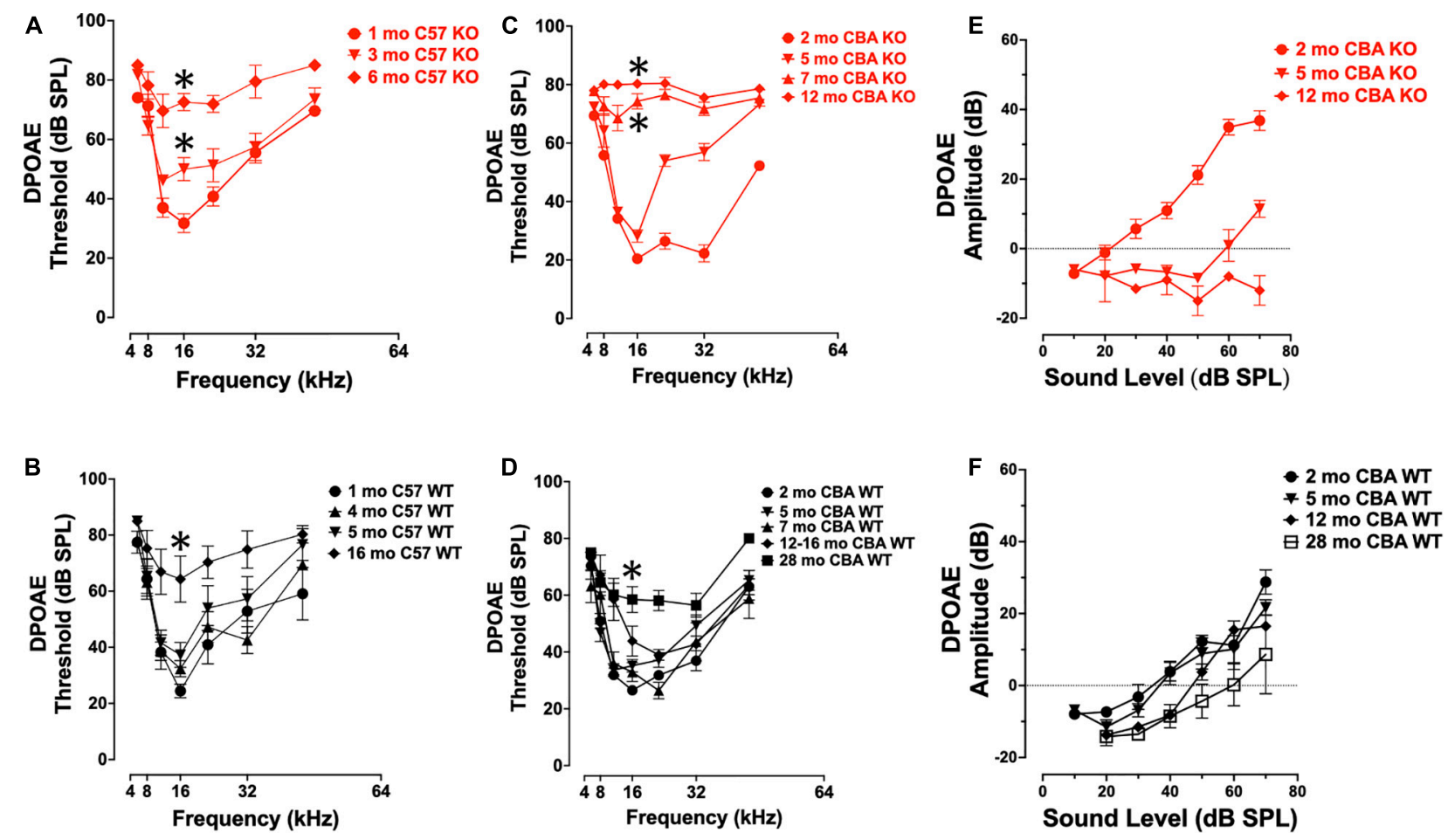

FIGURE 2 | Ocm KO mice show progressive elevation in distortion product otoacoustic emission (DPOAE) thresholds. (A,B) Mean \pm SEM DPOAE thresholds as a function of $f 2$ frequency for $1-6$ mo C57 KO $(\mathbf{A}, n=23)$ and $1-16$ mo WT mice $(\mathbf{B}, n=33)$. Significant $(p<0.05)$ total threshold shifts were observed when comparing from $1 \mathrm{mo}$ to all other ages and $3-6$ mo ages of C57 KO animals. Total threshold shifts were significantly different between 5 and $15-16$ mo in C57 WT. (C,D) Mean \pm SEM DPOAE thresholds as a function of $f 2$ frequency for CBA mice. (C) DPOAE thresholds from 2 to 12 mo CBA KO $(n=31)$ animals and (D) $2-28$ mo WT $(n=49)$ animals. Total threshold shifts were significantly $(p<0.0001)$ different between 2 and 5 mo, and between 5 and 7 mo CBA WT. Total threshold shifts were significantly $(p<0.01)$ different between 7 and $12-16$ mo, and between $12-16$ mo and 28 mo CBA KO. (E,F) Mean \pm SEM DPOAE input/output functions from panels $(\mathbf{E}) \mathrm{KO}$ and $(\mathbf{F}) \mathrm{WT}$ animals. Asterisks represent significance $(p<0.05)$ from 1 to $2 \mathrm{mo}$ at $16 \mathrm{kHz}$.

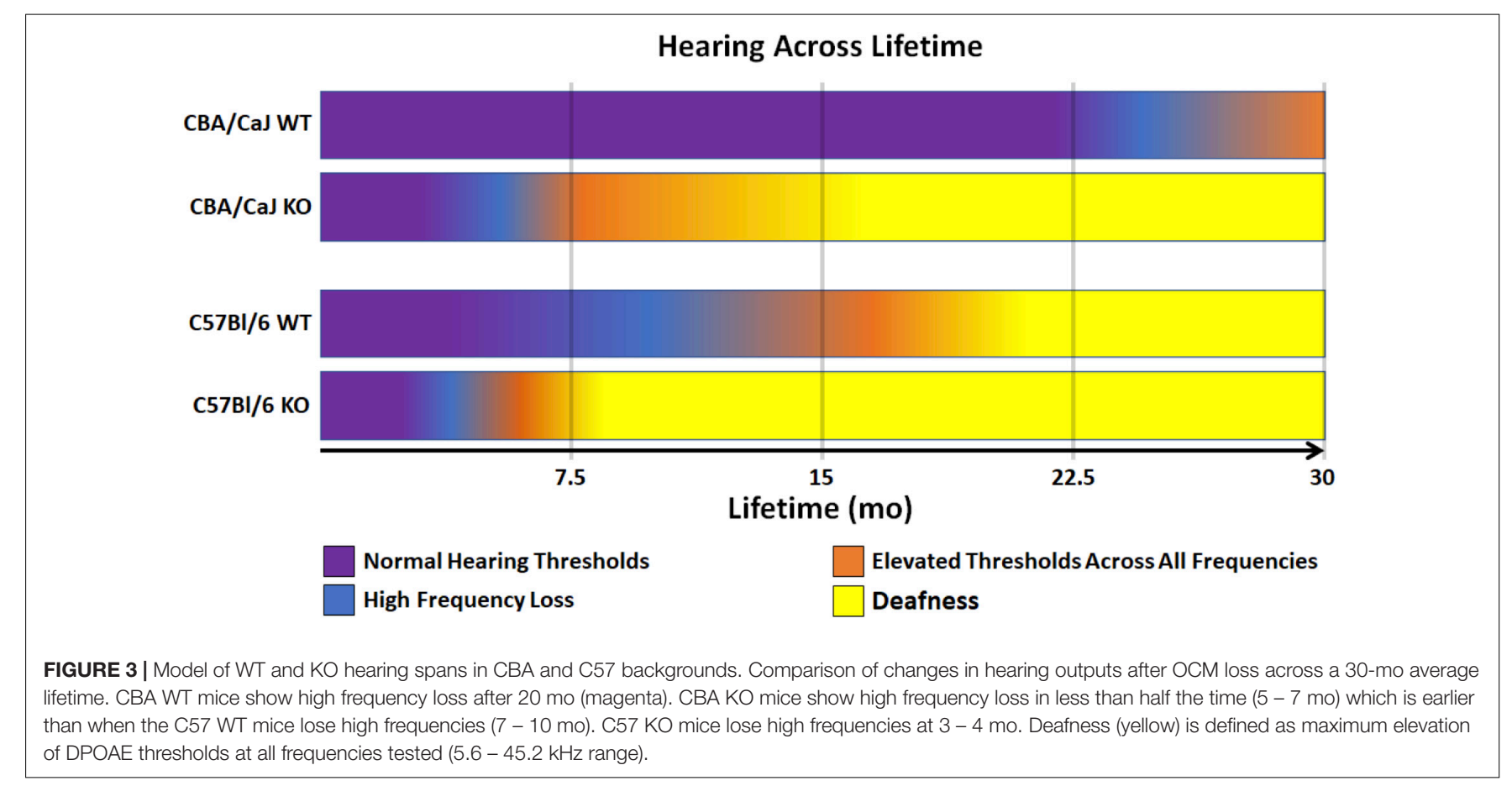


responsible for $\mathrm{OHC}$ electromotive function, though this too may be dysfunctional (Figures 4D,G, white). WT animals maintain robust $\mathrm{OHC}$ numbers throughout the first year of life and are only missing approximately $50 \%$ of the $\mathrm{OHCs}$ along the entire cochlear spiral by 30 mo (Figure 4I, top). By contrast, KO animals have lost the majority of OHCs by 12 mo (Figure 4I, top). Similar to our previous report (Tong et al., 2016), WT OHC numbers were maintained through 5 mo of age in the C57 background (Figures 4F-I) unlike Ocm KO animals (Figures 4H,I, bottom). Prestin was also abundant in the remaining OHCs of aged WT mice (Figures 4F,G) and KO OHCs at 5 - 6 mo (Figures 4D,H). Similar to the CBA WTs, OCM is expressed more intensely in the remaining $\mathrm{C} 57$ OHCs with age (Figure 4F) and across all frequencies (data not shown).

\section{Efferent Connections Are Lost With Age in Wild Type and KO Animals}

Synaptic dysfunction, rewiring, and $\mathrm{OHC}$ loss are common phenotypes of the aging cochlea. Activation of cholinergic medial olivocochlear (MOC) efferent axons protects $\mathrm{OHCs}$ from excessive noise and $\mathrm{Ca}^{2+}$ by modulating $\mathrm{OHCs}$ directly and altering their $\mathrm{Ca}^{2+}$-sensitive motility (Simmons, 2002; Maison et al., 2003; Frolenkov, 2006; Boero et al., 2020). Loss of MOC efferents could enhance the dysfunctional state of aging OHCs and thus result in increased hearing thresholds (Fu et al., 2010). Therefore, we looked for the presence and absence of ChAT-labeled efferent terminal clusters in aging WT and KO animals. In both CBA and C57 mice, we co-labeled surface preparations (Figures 5A,B,E,F) and mid-modiolar cochlear sections (Figures 5C,D, CBA only) with DAPI to stain the nuclei and ChAT to label efferent fibers and terminal clusters. In CBA WT and KO mice, ChAT-labeled clusters were abundant at 2 4 mo. However, in older WT and KO animals, we observed many instances of OHC loss and absence of ChAT labeling on remaining OHCs. Apical OHCs lose ChAT clusters by $12 \mathrm{mo}$ in WT animals (Figure 5A\#). At $30 \mathrm{mo}$ in the $8 \mathrm{kHz}$ region, the few remaining OHCs do not have ChAT-labeled clusters (Figure 5\# and Supplementary Figure 2E). The KOs show some hair cell loss and efferent loss as early as $2 \mathrm{mo}$ in the $32 \mathrm{kHz}$ region, but the most dramatic efferent loss occurs in both apical and basal regions by 12 mo (Figure $5 \mathbf{B}^{*} \#$ ). Both $\mathrm{KO} \mathrm{OHC}$ and efferent loss appear similar to recent data collected from young and old human temporal bones (Wu et al., 2019; Wu et al., 2020). The WT OHC damage and/or loss was more pronounced in apical regions while the $\mathrm{KO} \mathrm{OHC}$ loss was pronounced in basal regions.

In $\mathrm{C} 57$ mice, the pattern of $\mathrm{OHC}$ and efferent loss was more pronounced. The WT apical OHC and efferent loss was evident as early as 5 mo and by 15 mo both apical and basal OHCs and efferent clusters were mostly absent (Figure 5E). In the $\mathrm{KO}$ at $2 \mathrm{mo}$, we observe apical efferent loss and basal OHC loss (Figure 5F). At $5 \mathrm{mo}$, the majority of basal OHCs were absent and there were also no efferents clusters. Apical regions had less OHC and efferent loss. WT mice showed similar apical and basal OHC and efferent loss while the $\mathrm{KO}$ showed more $\mathrm{OHC}$ and efferent loss in basal regions. Though the WT animals showed signs of efferent loss in the apex compared with the $\mathrm{KO}$ at $5-7 \mathrm{mo}$, there was no significant difference in their ChAT numbers at either region (Supplementary Figure 2C). The greatest significant difference at these ages was in the basal regions of the KO (Supplementary Figure 2D).

\section{Outer Hair Cell Dysfunction Occurs Prior to Cell Death}

We analyzed the loss of OHCs and ChAT-labeled efferent connections in relation to specific DPOAE frequency regions in CBA KO animals (Figure 6). We co-labeled surface preparations with antibodies to prestin and ChAT (Figures 6A-F). Single animal (Figures 6A-H) and cohort (Figures 6I,J) data are shown. At 3mo, we found robust DPOAE responses at 16 and $22 \mathrm{kHz}$ (Figure $\mathbf{6 H}$, purple) with the majority of hair cells present except in the extreme base (Figure 6G, purple). By $7 \mathrm{mo}$, responses were near the measurement ceiling at these frequency regions (Figure $\mathbf{6 H}$, green) and yet there are still hair cells present, particularly at $16 \mathrm{kHz}$ regions (Figure 6G, green). Indeed, DPOAE responses were elevated near maximal sound levels across the entire cochlea at $7 \mathrm{mo}$, but the majority of hair cells in all three $\mathrm{OHC}$ rows are present in the $4-16 \mathrm{kHz}$ regions and prestin was present in the remaining cells. ChAT was also present in the majority of the remaining cells, though not all (Figures 6C,D\#). Efferent loss was progressive, with the greatest loss occurring by 7 mo in the middle and high frequency regions (Figure 6I). We also plotted the percent $\mathrm{OHC}$ present as a function of cochlear frequency (Figure 6J). Middle frequency regions of the cochlea show the least efferent loss. The greatest loss of efferent clusters occurs in the regions of greatest OHC loss. Apical regions of the cochlea also show a loss of efferent clusters with little OHC loss. Taken together, there is a complex relationship between $\mathrm{OHC}$ loss, efferent terminal loss and hearing loss across cochlear frequency regions. In agreement with previous literature, high frequency regions are the most susceptible to trauma.

\section{DISCUSSION}

In this study, we assessed the role of OCM, an EF-hand CaBP predominately expressed in OHCs, in ARHL using two different genetic strains of mice: $\mathrm{CBA} / \mathrm{CaJ}$ and $\mathrm{C} 57 \mathrm{Bl} / 6 \mathrm{~J}$. The CBA mouse has a substantially later onset of ARHL compared to the C57 mouse. First, we engineered an $\mathrm{Ocm}$ KO mouse using C57Bl/6J and then backcrossed the $\mathrm{Ocm} \mathrm{KO}$ onto CBA/CaJ. Compared to their WT controls, both $\mathrm{Ocm}$ KO strains demonstrated an earlier progressive elevation of hearing thresholds, loss of efferent synaptic contacts, and OHC loss. Our results in the $\mathrm{Ocm} \mathrm{KO}$ suggest that alterations in $\mathrm{Ca}^{2+}$ signaling lead to $\mathrm{OHC}$ dysfunction before loss of efferent synaptic contacts and/or OHC loss. Although we do not have direct evidence, our results suggest that changes in the presence of efferent terminals may occur prior to $\mathrm{OHC}$ loss. The WT and Ocm KO mice share common aging characteristics despite differences in their temporal progression of hearing loss. Figure 7 represents a simple model illustrating the 

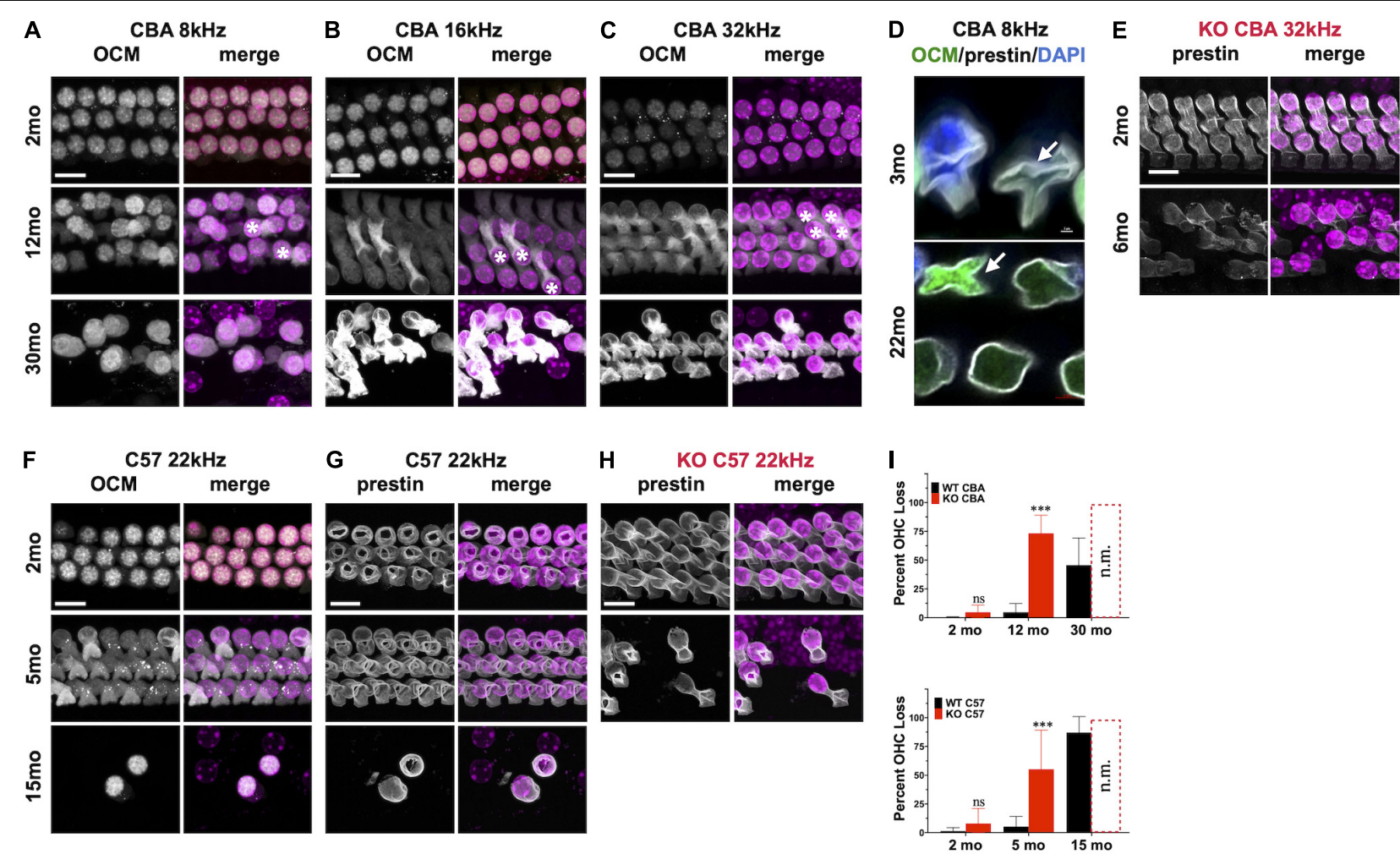

FIGURE 4 | Oncomodulin and Prestin are expressed in aged Outer hair cells (OHCs). (A-C) Surface preparations from 2 mo, 12 mo, and 30 mo CBA WT mice stained for OCM (gray) in the (A) $8 \mathrm{kHz}$, (B) $16 \mathrm{kHz}$, and (C) $32 \mathrm{kHz}$ regions. Asterisk represents enhanced OCM immunoreactivity. (D) Upper panel: 3 mo-old CBA/CaJ WT OHC at 8 kHz. Arrow indicates collapsed OHC with low OCM expression. Lower panel: 22 mo-old CBA CaJ at 8 kHz. Collapsed OHC with saturated OCM expression depicted by arrow. Stained with antibodies to OCM (green) and prestin (white) and co-stained with DAPI (blue). (E) 32 kHz region surface preparations from CBA KO mice stained for prestin at 2 mo and 6 mo. (F,G) $22 \mathrm{kHz}$ region surface preparations from 2 mo, 5 mo, and 15 mo C57 WT mice stained for panles (F) OCM and (G) prestin. (H) 22 kHz region surface preparations for C57 KO mice stained for prestin at 2 mo and 5 mo. (I) Upper panel: CBA WT and KO $\mathrm{OHC}$ loss quantified from surface preparations. Lower panel: C57 WT and KO OHC loss quantified from surface preparations. OHC loss was not measured from the KOs at 30 mo and 15 mo (n.m.). Loss was averaged from samples taken along the entire cochlear spiral. Error bars are S.D. of 3 samplings. Asterisks represent significance $(p<0.05)$ from aged-matched WT. n.s. is not significant.

general structural similarities between aged WT and young $\mathrm{Ocm}$ $\mathrm{KO}$ animals across frequencies. Both aged WT and young $\mathrm{KO}$ show OHCs and ChAT labeled efferent connections are lost with age. Importantly, even when ABR and DPOAE thresholds are highly elevated or not measurable across frequencies, the majority of OHCs and efferent terminals are still present in most frequency regions in both aged WT and younger Ocm KO animals, suggesting compromised $\mathrm{OHC}$ electromotility function. At least in OHCs, it is possible that disturbances in $\mathrm{Ca}^{2+}$ signaling caused by the removal of OCM lead to the early activation of a general aging process. The absence of OCM in OHCs appears to accelerate ARHL and mimics human presbycusis, which typically displays loss of nerve fibers and hair cells at apex and base (Wu et al., 2019). Future studies assessing both IHC and $\mathrm{OHC}$ survival and their neural connections in $\mathrm{Ocm} \mathrm{KO}$ animals will be essential to identifying other characteristics of human presbycusis.

\section{Extension of Previous Work}

Oncomodulin plays a major role in the maintenance of $\mathrm{OHC}$ intracellular $\mathrm{Ca}^{2+}$ levels. OHCs have very high levels of OCM with estimates reaching as high as $2-4 \mathrm{mM}$ suggesting that OCM provides the bulk of $\mathrm{Ca}^{2+}$ buffering in OHCs (Hackney et al., 2005). $\mathrm{Ca}^{2+}$ may be involved in regulating the motor capability underlying $\mathrm{OHC}$ cochlear amplification. The high concentration of OCM in OHCs, similar only to $\alpha \mathrm{PV}$ in skeletal muscle, may protect against deleterious consequences of $\mathrm{Ca}^{2+}$ loading after acoustic overstimulation. Thus, without OCM to buffer the $\mathrm{Ca}^{2+}$ levels in OHCs, cytoplasmic $\mathrm{Ca}^{2+}$ regulation could be severely disrupted and lead to $\mathrm{OHC}$ dysfunction and loss if nothing effectively compensates for its absence (Climer et al., 2019). To date, unlike previous genetic disruption studies of other mobile $\mathrm{Ca}^{2+}$ buffers that show little, if any, impact on hearing, OCM is the only known CaBP for which targeted deletion causes progressive hearing loss (Tong et al., 2016), suggesting the essential role of $\mathrm{Ca}^{2+}$ signaling in maintaining hearing health. Within the inner ear, targeted deletion of any of the other major EF-hand CaBPs (e.g., calbindin D28k, calretinin and $\alpha \mathrm{PV}$ ) show no hearing loss phenotype (Airaksinen et al., 2000; Schwaller et al., 2002). Using a targeted deletion of Ocm, Tong et al. (2016) demonstrated that the lack of OCM led to progressive cochlear dysfunction beginning after 1 mo and resulted in ABR threshold 


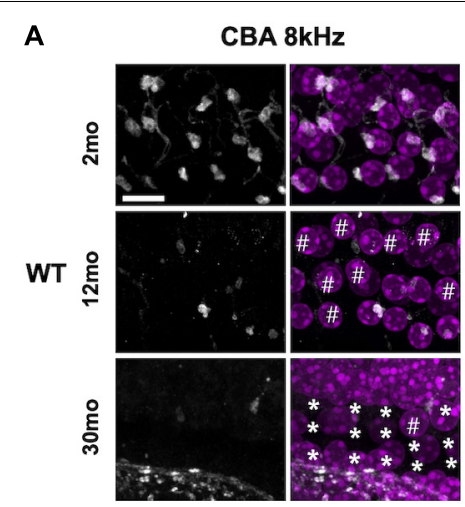

B

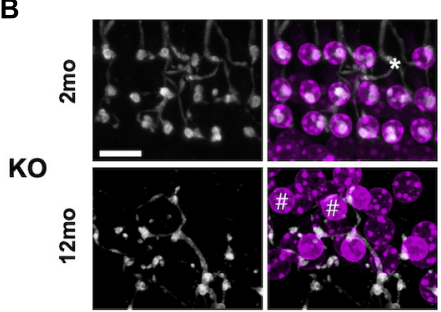

E

E $\quad$ C57 8kHz

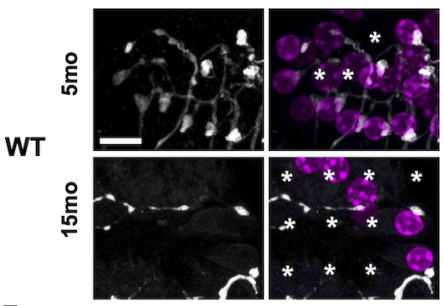

$\mathbf{F}$

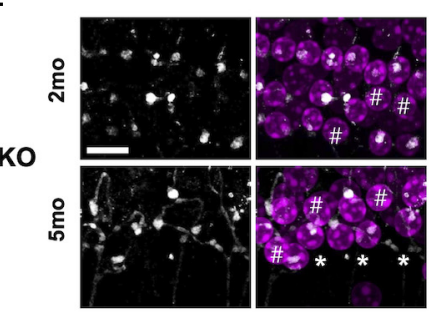

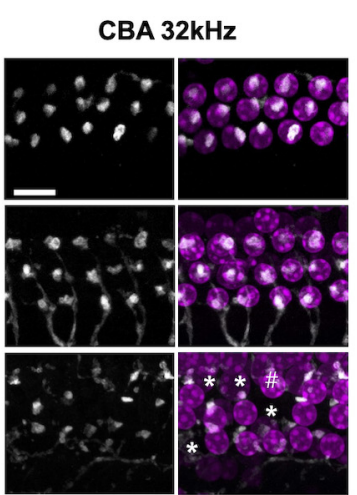

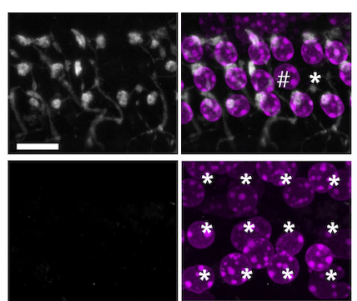

C57 32kHz
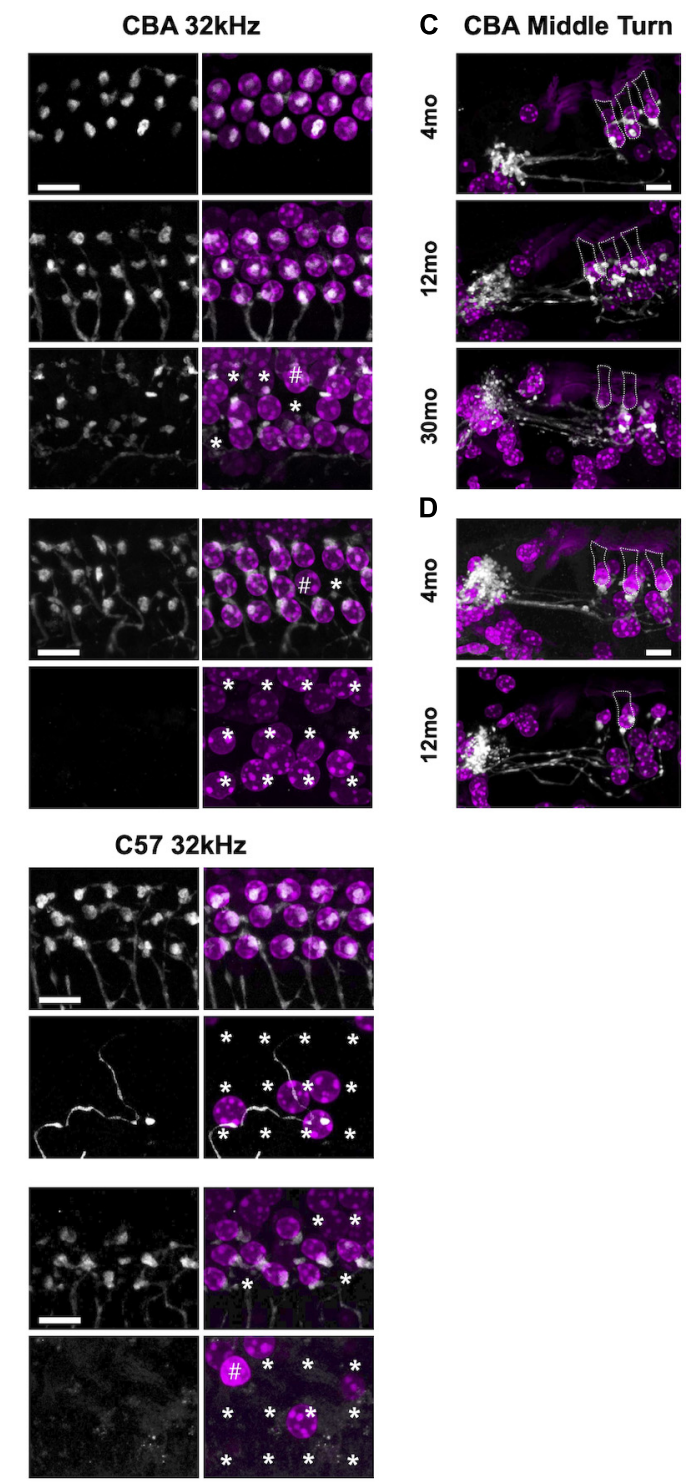

D

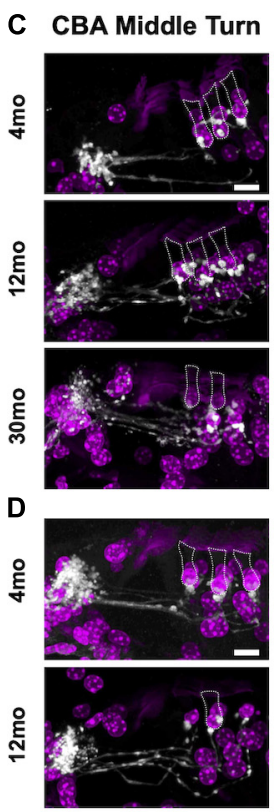

FIGURE 5 | Efferent clusters are lost at the extreme ends in aging WT and KO mice. Surface preparations showing choline acetyltransferase (ChAT)-labeled efferent clusters in the 8 and $32 \mathrm{kHz}$ regions of panel (A) CBA WT and (B) CBA KO mice. * Missing OHCs, \# OHCs without ChAT clusters. (C,D) Mid-modiolar sections of ChAT-labeled clusters in the middle turn of panel (E) CBA WT and (F) CBA KO mice. Dotted lines represent OHC borders from adjacent OHC in each row. Scale bars represent $10 \mu \mathrm{m}$.

shifts and loss of DPOAEs by $4-5$ mo of age. Functionally, the absence of OCM mimicked an accelerated aging or noise damage process. The presence of normal ABR and DPOAE thresholds in the $\mathrm{Ocm} \mathrm{KO}$ at 1 mo suggested that OCM was not essential for the development of cochlear function, but critically protects OHCs from damage in the adult ear. Similarly in the present study, we used a C57 mouse with germ-line deletion of OCM. The majority of C57 KO mice had significantly higher ABR and DPOAE threshold responses at $5-7$ mo across all frequencies, but the absence of responses could occur as early as 4 mo at some frequencies. Compared to the WT mice at $5 \mathrm{mo}$, these $\mathrm{Ocm}$ $\mathrm{KO}$ mice also exhibited increased $\mathrm{OHC}$ loss especially in basal regions and altered $\mathrm{OHC}$ prestin immunoreactivity. In fact, the
Ocm KO mice had hearing thresholds, OHC loss and prestin immunoreactivity comparable to 15 mo old WT C57 mice.

\section{Comparison of $\mathrm{Ocm} \mathrm{KO}$ on CBA With C57}

The two most utilized mouse models for studies of ARHL are the $\mathrm{C} 57 \mathrm{Bl} / 6 \mathrm{~J}$ and $\mathrm{CBA} / \mathrm{CaJ}$ strains. The $\mathrm{C} 57$ strain has a rapid, high-frequency hearing loss and loses most of its high-frequency hearing during the first $12 \mathrm{mo}$. In contrast, the CBA strain loses its hearing slowly with age. At early adult ages from 1 to 2 mo, functional measures of hearing for these two mouse strains are virtually indistinguishable. In the present study, the CBA 

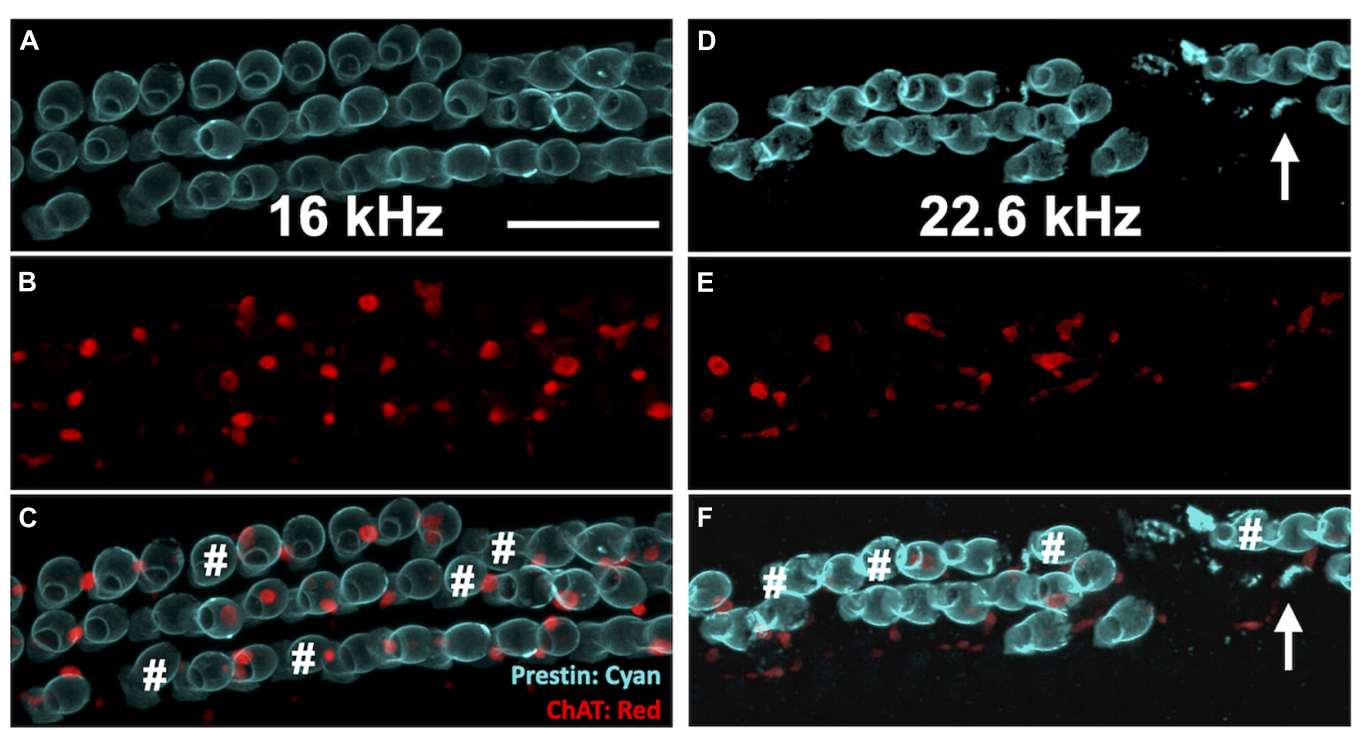

G
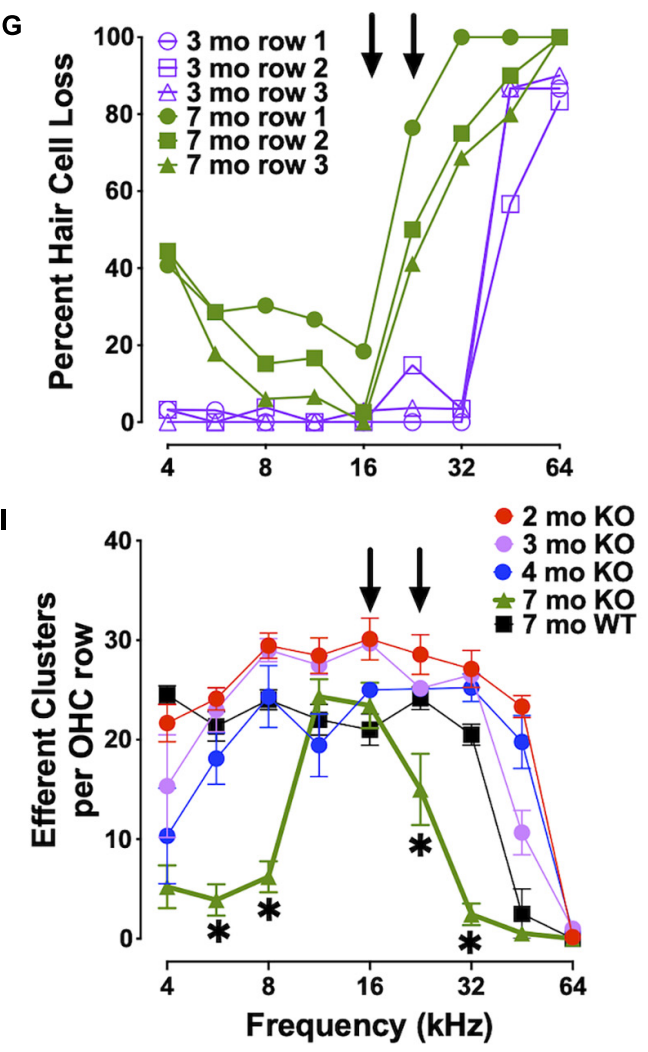

H
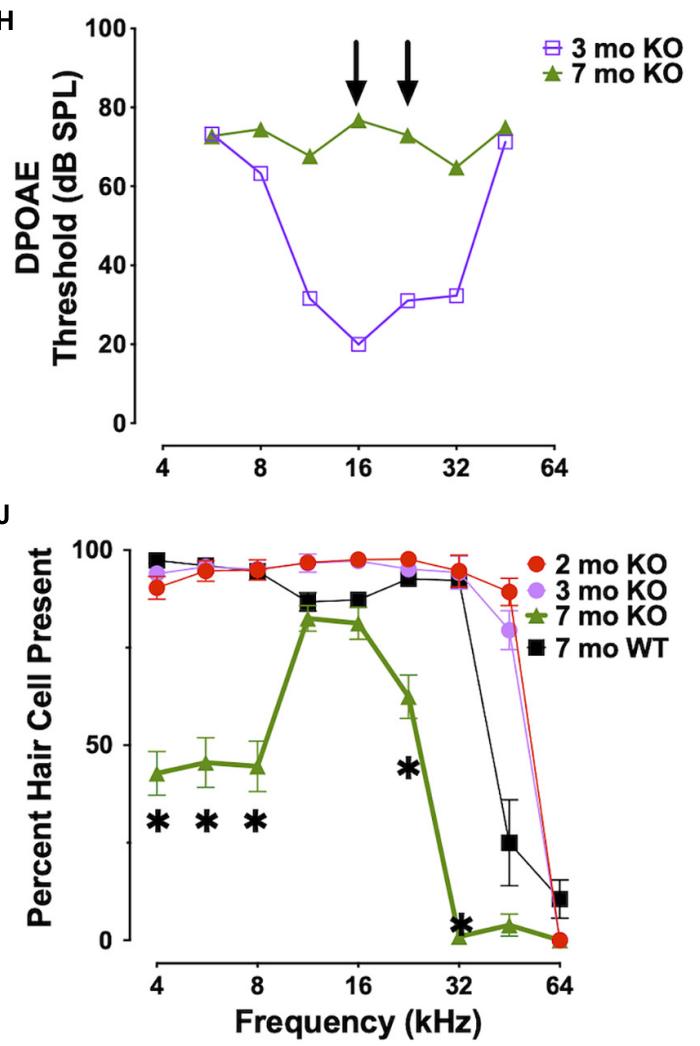

FIGURE 6 | Elevated thresholds occur even when OHCs and efferent clusters are present. (A-F) Surface preparations of a 7 mo CBA KO cochlea co-labeled with prestin (teal) and ChAT (red) in the 16 and $22 \mathrm{kHz}$ regions of CBA KO mice. \# OHCs without ChAT clusters. Arrows point to OHC fragments. Quantification of this 7 mo is shown in panels (G) and (H). (G)\% of OHCs missing from each OHC row from a single 3 mo and 7 mo CBA KO animal. Arrows point to 16 and $22 \mathrm{kHz}$ frequencies. (H) DPOAE thresholds of $3 \mathrm{mo}$ and $7 \mathrm{mo}$ from panel (G). Arrows point to 16 and $22 \mathrm{kHz}$ frequencies. (I) Quantification of the number of ChAT-labeled efferent clusters from CBA KO animals from 2 to $7 \mathrm{mo}(n=3 /$ age). (J) Quantification of the number of OHCs from CBA KO animals from 2 to 7 mo ( $n=3 /$ age). Scale represents $40 \mu \mathrm{m}$. Asterisks represent significance $(p<0.05)$ from $2 \mathrm{mo}$ at 16 and $22 \mathrm{kHz}$.

Ocm mutant mice present with an early onset ARHL as seen in the C57 WT and KO mice. Our CBA Ocm mutants, beginning around $5-7 \mathrm{mo}$, and aged WT mice show similar hallmarks of ARHL: (1) progressive elevation of DPOAE thresholds, (2) loss of OHC efferent terminals, and (3) OHC loss. In Ocm KOs on a CBA background, our data show a progressive elevation 

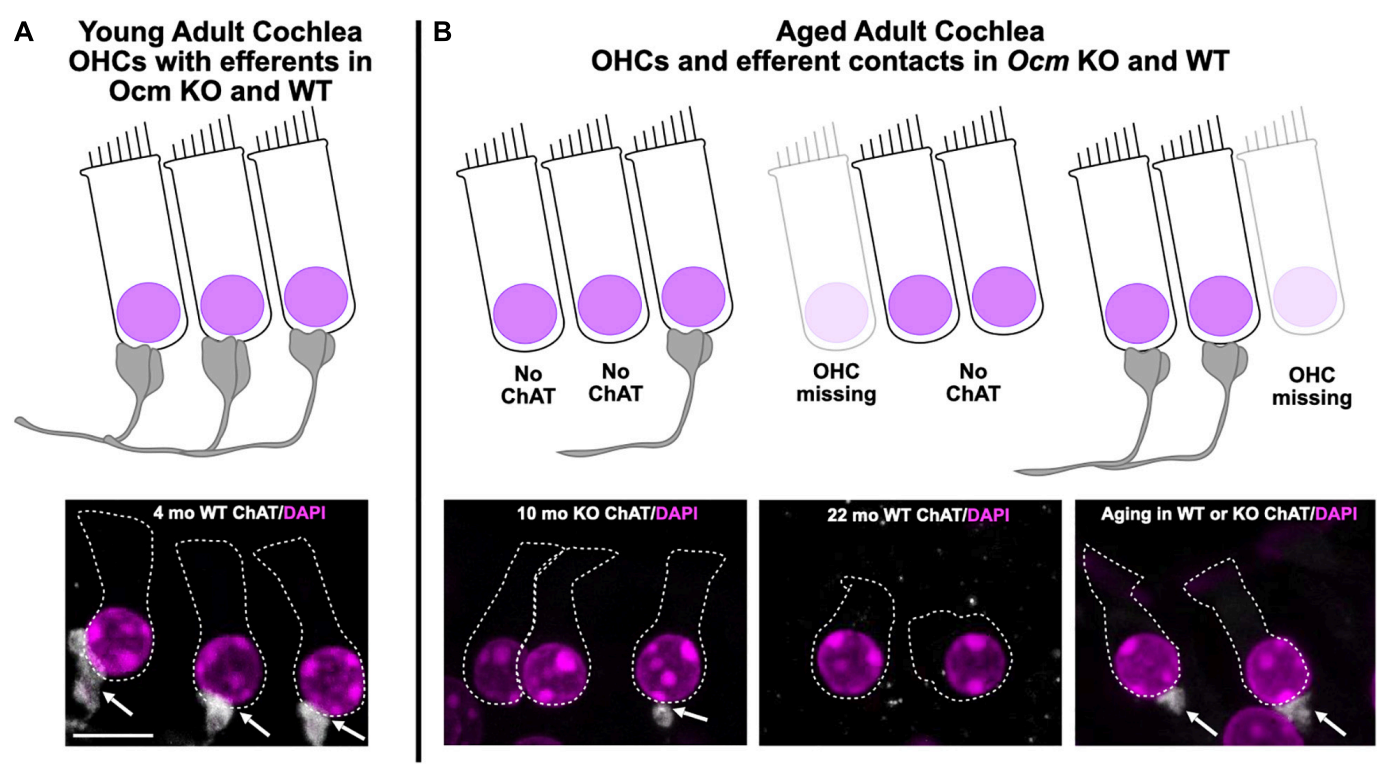

FIGURE 7 | Model of common Ocm KO and aging characteristics. (A) Young adult CBA WT and KO animals have organized OHC rows with efferent clusters present on all cells. (B) During aging, OHCs are lost and not all of the remaining cells will possess efferent synaptic connections. In the WT animals, this occurs after 1 year, but the KOs show efferent and $\mathrm{OHC}$ loss much earlier. This demonstrates that dysregulated $\mathrm{Ca}^{2+}$ signaling can enhance the effects of aging. Scale bar $=10 \mu \mathrm{m}$.

of DPOAE thresholds over 12 mo. In contrast, WT hearing thresholds and response magnitudes change little from 2 to 12 mo. Similar to our observations in the C57 strain, young (2 mo) CBA Ocm KO mice had DPOAE thresholds nearly identical to WT controls at lower frequencies and somewhat enhanced at higher frequencies. In older $\mathrm{CBA} O \mathrm{~cm} \mathrm{KO}$ mice, maximum DPOAE threshold shifts occurred at frequencies $16 \mathrm{kHz}$ and higher, which is within the region of greatest hearing sensitivity in these animals. $\mathrm{Ocm} \mathrm{KO}$ mice had significantly higher thresholds at all measured frequencies between 5 and 7 mo. At 12 mo, DPOAE thresholds were only slightly elevated in WT control mice and were not measurable in the $\mathrm{Ocm}$ KO. In the present study, WT CBA mice had measurable DPOAEs up to 28 mo and we did not find significant ARHL until after 28 mo. These results suggest that $\mathrm{Ocm}$ deletion in CBA mice leads to an accelerated ARHL phenotype that is faster than the WT C57, but more delayed than in C57 KO mice. Although the specific temporal pattern of hearing loss differs, the results were similar to our previous report and validate that OCM expression is critical for either the maintenance of cochlear function and/or protecting OHCs from damage as previously hypothesized (Tong et al., 2016). Further, having a similar early progression of hearing loss across genetic strains argues that OCM possibly mediates sensitivity to ARHL. As illustrated in Figure 3, OCM deletion reduces the period of measurable hearing thresholds by over $50 \%$ in CBA and C57 mice.

\section{Comparison of Ocm KO With Age-Related Hearing Loss}

In $\mathrm{Ocm}$ KO mice with high DPOAE thresholds, the majority of OHCs are still present. Although dysregulated $\mathrm{Ca}^{2+}$ signaling is a known contributor to apoptotic cell death, OHC loss occurs well after the loss of DPOAEs in the $\mathrm{Ocm}$ KO mouse. Since DPOAE responses are a direct measure of $\mathrm{OHC}$ function, elevated DPOAE thresholds suggest the remaining $\mathrm{OHCs}$ in the Ocm KO must be either "silent" or unresponsive to sounds. In CBA mutants, supra threshold DPOAE responses show rapid attenuation between 2 and 5 mo. At $32 \mathrm{kHz}$, 2 mo sensitivity to sounds deteriorated nearly $40 \mathrm{~dB}$ by 5 mo even though the majority of OHCs were still present. The lack of DPOAEs might suggest a decrease in expression of the motor protein prestin, as loss of prestin is correlated with elevated DPs (Liberman et al., 2002). However, this is unlikely due to the robust prestin immunoreactivity in these unresponsive OHCs. The lack of DPOAEs but presence of prestin protein would suggest that $\mathrm{Ca}^{2+}$ dysregulation makes OHCs dysfunctional in other ways such as in their biophysical characteristics. Recent studies of aged OHCs are consistent with the idea that OHCs undergo a period of dysfunction prior to OHC loss. In a study of aged OHCs from early onset and late onset ARHL mouse models, Jeng et al. (2021) suggested that age-related $\mathrm{OHC}$ dysfunction is not due to apoptosis. They primarily focused their studies on OHCs from the $9-12 \mathrm{kHz}$ region. At 12 - 13 mo irrespective of whether OHCs were from early and late ARHL mice, they found $\mathrm{OHCs}$ had similar biophysical properties related to their peak current-voltage relationships, potassium currents, membrane capacitance, and membrane voltage measurements. With the exception of membrane voltage, they showed that these biophysical properties decreased similarly with age, being highest in the youngest animals. In addition to OHC loss, they found decreases in $\mathrm{OHC}$ size as indicated by decreases in membrane capacitance, $\mathrm{OHC}$ ribbons, and the mRNA expression 
of Slc26a5 and Ocm (Jeng et al., 2021). Most, if not all, of these features changed similarly, independent of the ARHL onset suggesting that they are general features of aging in $\mathrm{OHCs}$, and not necessarily related to the $\mathrm{OHC}$ dysfunction observed in ARHL. Thus, it is possible that OHC-dependent ARHL is associated with disruption of cellular processes of transduction or motility. In the present study, we observed both prestin and OCM immunoreactivity in aged WT C57 and CBA OHCs (Figure 4). However, both proteins label more intensely with age (Figure 4), most likely either due to changes in cellular volume or expression. The seemingly enhanced expression of OCM could simply represent an artifact of shrunken $\mathrm{OHC}$ volume or it might convey that there is increased $\mathrm{Ca}^{2+}$ activity. In this regard, altered $\mathrm{Ca}^{2+}$ buffering could change the dynamics of organelles (e.g., mitochondria and endoplasmic reticulum), which store and release $\mathrm{Ca}^{2+}$ as well as alter ATP utilization. Although it is possible the overall mRNA expression of $\mathrm{Ocm}$ and Slc26a5 (prestin) in the cochlea decreases with age as reported by Jeng et al. (2020b), the ramifications for protein levels remain unclear. A more relevant question is whether their functions are somehow compromised. Jeng et al. (2020b) measured nonlinear capacitance in aged OHCs and found that it decreased, but when normalized, the decrease appears independent from an ARHL phenotype. It is possible that smaller cell volumes due to an age-related shrinkage causes a relative increase in prestin protein levels. A tangential question is raised: though prestin is still present in aged OHCs, how much function does it retain? Overexpression of dysfunctional prestin in the mutant knock-in is more deadly to OHCs than complete lack of prestin suggesting features associated with prestin such as electromotility and axial stiffness could be contributors to OHC silencing during ARHL (Dallos et al., 1997; He et al., 2003; Keller et al., 2014). Our finding that OCM localization appears to undergo changes associated with age in WT mice might indicate age-associated changes in $\mathrm{Ca}^{2+}$ activity. It seems one of our challenges will be separating general features of aging in $\mathrm{OHCs}$ from those that lead to ARHL.

\section{Outer Hair Cell Efferent Innervation During Age-Related Hearing Loss}

Sound- or electrically induced activity of the cholinergic MOC pathway suppresses OHC responses leading to reduced cochlear output and protection from acoustic injury (Rajan and Johnstone, 1989; Zheng et al., 1997; Maison and Liberman, 2000). Previous studies have shown that the function of the MOC system declines with age prior to OHC degeneration in both humans and mice (Sun and Kim, 1999; Kim et al., 2002; Jacobson et al., 2003; Zhu et al., 2007; Boero et al., 2020). In adult mice, OHCs are primarily innervated by the cholinergic MOC neurons, which modulate amplification of the cochlear partition (Guinan, 2006). At MOC - OHC synapses, release of acetylcholine (ACh) causes $\mathrm{Ca}^{2+}$ entry through $\alpha 9 \alpha 10$ nicotinic $\mathrm{ACh}$ receptors $(\alpha 9 \alpha 10 \mathrm{nAChRs})$. The $\mathrm{Ca}^{2+}$ influx activates a hyperpolarizing current through $\mathrm{Ca}^{2+}$-activated small conductance $\mathrm{K}^{+}$(SK2) channels on the OHC and causes OHCs to elongate (Wersinger and Fuchs, 2011; Guinan, 2018; Fuchs and Lauer, 2019). The overall effect of OHC elongation is believed to be protective (Frolenkov, 2006; Ciganovic et al., 2018). In both our Ocm KO and in aged WT controls, the presence of cholinergic efferent terminals contacting OHCs decreased in both low and high frequency regions with age (Figure 5). Jeng et al. (2021) also investigated whether the efferent innervation was retained in the aged apical cochlea of early and late onset ARHL mice. Although Jeng et al. (2020b) found no significant change in either the percentage of OHCs with SK2 immunoreactivity or the percentage of SK2 puncta juxtaposed to cholinergic terminals, they did note that some apical OHCs showed only efferent terminals or SK2 puncta. It has been known for some time that a reduction in OAEs correlates with impaired cochlear responses (de la Cruz et al., 1998; Zhu et al., 2007). If efferent terminals or SK2 puncta are lost, OHCs would lose that protection and have increased susceptibility to noise damage. MOC-mediated resistance to ARHL has been induced by enhancing $\alpha 9 \alpha 10 \mathrm{nAChR}$ complexes on OHCs (Boero et al., 2020). Thus, the efferent loss or decline during aging could exacerbate ARHL, particularly in regard to discriminating between different stimuli under sundry conditions (Kobrina et al., 2020). Future studies should investigate the time course of efferent synaptic loss on the pre- and post-synaptic $\mathrm{OHC}$ junction.

\section{CONCLUSION}

By comparing loss of OCM in two mouse strains with contrasting temporal patterns of ARHL, we have demonstrated that OCM and $\mathrm{Ca}^{2+}$ buffering play an important role in regulating ARHL. This phenomenon leads us to speculate that $\mathrm{Ca}^{2+}$ signaling may be a gateway into a global process that regulates $\mathrm{OHC}$ functionality and response to stimuli. The process breaks down during aging leading to silent or possibly senescent OHCs (elevated DPOAEs), loss of efferent synapses, OHC loss, and hearing loss. Likely, there are multiple avenues for disrupting this aging process, which leads to the differences in ARHL progression that we see across animal models. By interfering with $\mathrm{Ca}^{2+}$ signaling, which is fundamental to many intracellular processes, we were able to expose the process and enhance its destabilization.

\section{DATA AVAILABILITY STATEMENT}

The raw data supporting the conclusions of this article will be made available by the authors, without undue reservation.

\section{ETHICS STATEMENT}

The animal study was reviewed and approved by the Baylor University Institutional Animal Care and Use Committee. 


\section{AUTHOR CONTRIBUTIONS}

LC and DS wrote the manuscript. LC, AH, KM, AC, AL, and PS performed the tissue preparation, immunostaining, and imaging. LC, DS, AH, YY, AC, and AL contributed to ABR and DPOAE analysis. All authors discussed and edited the manuscript. All authors contributed to the article and approved the submitted version.

\section{FUNDING}

This research was supported by the following grants to DS: National Institute on Deafness and Other Communication Disorders (K18 DC013304), a 2015-2016 Fulbright Scholar Award, and an American Hearing Research Foundation grant.

\section{ACKNOWLEDGMENTS}

We would like to thank Jack Charles, Jemima McCluskey, Adel Azghadi, Joyce Tsang, and Maria Kazantsev for technical assistance.

\section{REFERENCES}

Airaksinen, L., Virkkala, J., Aarnisalo, A., Meyer, M., Ylikoski, J., and Airaksinen, M. S. (2000). Lack of calbindin-D28k does not affect hearing level or survival of hair cells in acoustic trauma. ORL J. Otorhinolaryngol. Relat. Spec. 62, 9-12. doi: $10.1159 / 000027708$

Altschuler, R. A., Dolan, D. F., Halsey, K., Kanicki, A., Deng, N., Martin, C., et al. (2015). Age-related changes in auditory nerve-inner hair cell connections, hair cell numbers, auditory brain stem response and gap detection in UM-HET4 mice. Neuroscience 292, 22-33. doi: 10.1016/j.neuroscience.2015.01.068

Bielefeld, E. C., Tanaka, C., Chen, G. D., and Henderson, D. (2010). Age-related hearing loss: is it a preventable condition? Hear. Res. 264, 98-107. doi: 10.1016/ j.heares.2009.09.001

Boero, L. E., Castagna, V. C., Terreros, G., Moglie, M. J., Silva, S., Maass, J. C., et al. (2020). Preventing presbycusis in mice with enhanced medial olivocochlear feedback. Proc. Natl. Acad. Sci. U.S.A. 117, 11811-11819. doi: 10.1073/pnas. 2000760117

Chen, G. D., Li, M., Tanaka, C., Bielefeld, E. C., Hu, B. H., Kermany, M. H., et al. (2009). Aging outer hair cells (OHCs) in the Fischer 344 rat cochlea: function and morphology. Hear. Res. 248, 39-47. doi: 10.1016/j.heares.2008.11.010

Ciganovic, N., Warren, R. L., Keceli, B., Jacob, S., Fridberger, A., and Reichenbach, T. (2018). Static length changes of cochlear outer hair cells can tune lowfrequency hearing. PLoS Comput. Biol. 14:e1005936. doi: 10.1371/journal.pcbi. 1005936

Climer, L. K., Cox, A. M., Reynolds, T. J., and Simmons, D. D. (2019). Oncomodulin: the enigmatic parvalbumin protein. Front. Mol. Neurosci. 12:235. doi: $10.3389 /$ fnmol.2019.00235

Cruickshanks, K. J., Wiley, T. L., Tweed, T. S., Klein, B. E., Klein, R., MaresPerlman, J. A., et al. (1998). Prevalence of hearing loss in older adults in Beaver Dam, Wisconsin. The epidemiology of hearing loss study. Am. J. Epidemiol. 148, 879-886. doi: 10.1093/oxfordjournals.aje.a009713

Dallos, P., He, D. Z., Lin, X., Sziklai, I., Mehta, S., and Evans, B. N. (1997). Acetylcholine, outer hair cell electromotility, and the cochlear amplifier. J. Neurosci. 17, 2212-2226. doi: 10.1523/jneurosci.17-06-02212.1997

de la Cruz, R. R., Pastor, A. M., Martinez-Guijarro, F. J., Lopez-Garcia, C., and Delgado-Garcia, J. M. (1998). Localization of parvalbumin, calretinin, and calbindin D-28k in identified extraocular motoneurons and internuclear

\section{SUPPLEMENTARY MATERIAL}

The Supplementary Material for this article can be found online at: https://www.frontiersin.org/articles/10.3389/fnagi. 2021.749729/full\#supplementary-material

Supplementary Figure 1 | (A) 360 bp PCR product for 12 CBA Ocm KO animals (lanes 2-13) and 1 CBA/CaJ control (lane 14). The DNA ladder is shown in lane 1. (B) Chromatograph of sequencing results from lane 2 and lane 14 showing that neither KO nor control animals have the G- > A Cdh23 ${ }^{\text {ahl }}$ mutation. (C,D) 5 mo old CBA wild type (WT) (C) and CBA Ocm KO (D) mid-modiolar sections stained with antibodies to Oncomodulin (OCM) (gray). Co-stained with phalloidin (green) and DAPI (magenta). Scale bar $=10 \mu \mathrm{m}$. (E,F) Line profile of $1 \mathrm{mo}$ old $(\mathbf{E})$ and 20 mo old (F) CBA WT mid-modiolar section stained with antibodies to OCM (gray) and co-stained with DAPI (magenta).

Supplementary Figure 2 | Cytoplasmic vs. nuclear OCM fluorescence during aging in WT (A) CBA and (B) C57 mice. (A) 8 and $32 \mathrm{kHz} \mathrm{c} / \mathrm{n}$ ratios from region of interests (ROls) at $1-2 \mathrm{mo}, 12 \mathrm{mo}$, and 20-30 mo $(n=5)$ CBA WT mice. (B) $8 \mathrm{kHz}$ and $22 \mathrm{kHz} \mathrm{c} / \mathrm{n}$ ratios from ROls at $1-2 \mathrm{mo}(n=2)$ and $5-7 \mathrm{mo}(n=2)$ in C57 WT mice. Quantification of choline acetyltransferase (ChAT) labeled efferent clusters in the $5-8 \mathrm{kHz}$ and $22-40 \mathrm{kHz}$ regions of panels (C) C57 WT and (D) C57 KO. No statistical difference in ChAT clusters between any C57 WT regions. All WT regions were statistically significant $(p<0.5)$ from basal region of $5-7 \mathrm{mo}$ $\mathrm{KO}$. (E) ChAT clusters from the 8 and $22-45 \mathrm{kHz}$ regions of WT CBA mice at 1 $2 \mathrm{mo}, 12 \mathrm{mo}(n=2)$ and $28 \mathrm{mo}(n=2) .3$ cochlea per condition unless otherwise indicated. All asterisks indicate $p<0.5$.

neurons of the cat. J. Comp. Neurol. 390, 377-391. doi: 10.1002/(sici)10969861(19980119)390:3\&lt;377::aid-cne6\&gt;3.0.co;2-z

Fettiplace, R., and Nam, J. H. (2019). Tonotopy in calcium homeostasis and vulnerability of cochlear hair cells. Hear. Res. 376, 11-21. doi: 10.1016/j.heares. 2018.11.002

Francis, H. W., Ryugo, D. K., Gorelikow, M. J., Prosen, C. A., and May, B. J. (2003). The functional age of hearing loss in a mouse model of presbycusis. II. Neuroanatomical correlates. Hear. Res. 183, 29-36. doi: 10.1016/s03785955(03)00212-0

Fridberger, A., Flock, A., Ulfendahl, M., and Flock, B. (1998). Acoustic overstimulation increases outer hair cell $\mathrm{Ca} 2+$ concentrations and causes dynamic contractions of the hearing organ. Proc. Natl. Acad. Sci. U.S.A. 95, 7127-7132. doi: 10.1073/pnas.95.12.7127

Frisina, R. D. (2009). Age-related hearing loss: ear and brain mechanisms. Ann. N. Y. Acad. Sci. 1170, 708-717. doi: 10.1111/j.1749-6632.2009.03 931.x

Frisina, R. D., Singh, A., Bak, M., Bozorg, S., Seth, R., and Zhu, X. (2011). F1 (CBAxC57) mice show superior hearing in old age relative to their parental strains: hybrid vigor or a new animal model for golden ears? Neurobiol. Aging 32, 1716-1724. doi: 10.1016/j.neurobiolaging.2009. 09.009

Frolenkov, G. I. (2006). Regulation of electromotility in the cochlear outer hair cell. J. Physiol. 576(Pt 1), 43-48. doi: 10.1113/jphysiol.2006.114975

Fu, B., Le Prell, C., Simmons, D., Lei, D., Schrader, A., Chen, A. B., et al. (2010). Age-related synaptic loss of the medial olivocochlear efferent innervation. Mol. Neurodegener. 5:53.

Fuchs, P. A., and Lauer, A. M. (2019). Efferent inhibition of the cochlea. Cold Spring Harb. Perspect. Med. 9:a033530. doi: 10.1101/cshperspect.a03 3530

Furness, D. N., Mahendrasingam, S., Ohashi, M., Fettiplace, R., and Hackney, C. M. (2008). The dimensions and composition of stereociliary rootlets in mammalian cochlear hair cells: comparison between high- and low-frequency cells and evidence for a connection to the lateral membrane. J. Neurosci. 28, 6342-6353. doi: 10.1523/JNEUROSCI.1154-08.2008

Greenwood, D. D. (1990). A cochlear frequency-position function for several species-29 years later. J. Acoust. Soc. Am. 87, 2592-2605. doi: 10.1121/1. 399052 
Guinan, J. J. Jr. (2006). Olivocochlear efferents: anatomy, physiology, function, and the measurement of efferent effects in humans. Ear. Hear. 27, 589-607. doi: 10.1097/01.aud.0000240507.83072.e7

Guinan, J. J. Jr. (2018). Olivocochlear efferents: their action, effects, measurement and uses, and the impact of the new conception of cochlear mechanical responses. Hear. Res. 362, 38-47. doi: 10.1016/j.heares.2017.12.012

Hackney, C. M., Mahendrasingam, S., Penn, A., and Fettiplace, R. (2005). The concentrations of calcium buffering proteins in mammalian cochlear hair cells. J. Neurosci. 25, 7867-7875. doi: 10.1523/ineurosci.1196-05.2005

He, D. Z., Jia, S., and Dallos, P. (2003). Prestin and the dynamic stiffness of cochlear outer hair cells. J. Neurosci. 23, 9089-9096. doi: 10.1523/jneurosci.23-27-09089. 2003

Henry, K., and Chole, R. (1980). Genotypic differences in behavioral, physiological and anatomical expressions of age-related hearing loss in the laboratory mouse. Audiology 19, 369-383. doi: 10.3109/00206098009070071

Henry, K. R., and Lepkowski, C. M. (1978). Evoked potential correlates of genetic progressive hearing loss:age-related changes from the ear to the inferior colliculus ofC57BL/6 and CBA/J mice. Acta Oto-Laryngol. 86, 366-374. doi: $10.3109 / 00016487809107515$

Henzl, M. T., Shibasaki, O., Comegys, T. H., Thalmann, I., and Thalmann, R. (1997). Oncomodulin is abundant in the organ of Corti. Hear. Res. 106, 105-111. doi: 10.1016/s0378-5955(97)00005-1

Hu, B. H., Henderson, D., and Nicotera, T. M. (2006). Extremely rapid induction of outer hair cell apoptosis in the chinchilla cochlea following exposure to impulse noise. Hear. Res. 211, 16-25. doi: 10.1016/j.heares.2005.08.006

Jacobson, M., Kim, S., Romney, J., Zhu, X., and Frisina, R. D. (2003). Contralateral suppression of distortion-product otoacoustic emissions declines with age: a comparison of findings in CBA mice with human listeners. Laryngoscope 113, 1707-1713. doi: 10.1097/00005537-200310000-00009

Jaramillo, F. (1995). Signal transduction in hair cells and its regulation by calcium. Neuron 15, 1227-1230. doi: 10.1016/0896-6273(95)90003-9

Jeng, J. Y., Carlton, A. J., Johnson, S. L., Brown, S. D. M., Holley, M. C., Bowl, M. R., et al. (2021). Biophysical and morphological changes in inner hair cells and their efferent innervation in the ageing mouse cochlea. J. Physiol. 599, 269-287. doi: 10.1113/JP280256

Jeng, J. Y., Ceriani, F., Olt, J., Brown, S. D. M., Holley, M. C., Bowl, M. R., et al. (2020a). Pathophysiological changes in inner hair cell ribbon synapses in the ageing mammalian cochlea. J. Physiol. 598, 4339-4355. doi: 10.1113/JP280018

Jeng, J. Y., Johnson, S. L., Carlton, A. J., De Tomasi, L., Goodyear, R. J., De Faveri, F., et al. (2020b). Age-related changes in the biophysical and morphological characteristics of mouse cochlear outer hair cells. J. Physiol. 598, 3891-3910. doi: 10.1113/JP279795

Jimenez, A. M., Stagner, B. B., Martin, G. K., and Lonsbury-Martin, B. L. (1999). Age-related loss of distortion product otoacoustic emissions in four mouse strains. Hear. Res. 138, 91-105. doi: 10.1016/s0378-5955(99)00154-9

Keller, J. P., Homma, K., Duan, C., Zheng, J., Cheatham, M. A., and Dallos, P. (2014). Functional regulation of the SLC26-family protein prestin by calcium/calmodulin. J. Neurosci. 34, 1325-1332. doi: 10.1523/jneurosci.402013.2014

Kim, S., Frisina, D. R., and Frisina, R. D. (2002). Effects of age on contralateral suppression of distortion product otoacoustic emissions in human listeners with normal hearing. Audiol. Neurootol. 7, 348-357. doi: 10.1159/000066159

Kobrina, A., Schrode, K. M., Screven, L. A., Javaid, H., Weinberg, M. M., Brown, G., et al. (2020). Linking anatomical and physiological markers of auditory system degeneration with behavioral hearing assessments in a mouse (Mus musculus) model of age-related hearing loss. Neurobiol. Aging 96, 87-103. doi: 10.1016/j.neurobiolaging.2020.08.012

Lauer, A. M., Fuchs, P. A., Ryugo, D. K., and Francis, H. W. (2012). Efferent synapses return to inner hair cells in the aging cochlea. Neurobiol. Aging 33, 2892-2902. doi: 10.1016/j.neurobiolaging.2012.02.007

Lenzi, D., and Roberts, W. M. (1994). Calcium signalling in hair cells: multiple roles in a compact cell. Curr. Opin. Neurobiol. 4, 496-502. doi: 10.1016/09594388(94)90049-3

Li, H. S., and Borg, E. (1991). Age-related loss of auditory sensitivity in two mouse genotypes. Acta Otolaryngol. 111, 827-834. doi: 10.3109/00016489109138418

Li, H. S., and Hultcrantz, M. (1994). Age-related degeneration of the organ of corti in two genotypes of mice. ORL 56, 61-67. doi: 10.1159/000276611
Liberman, M. C., Gao, J., He, D. Z., Wu, X., Jia, S., and Zuo, J. (2002). Prestin is required for electromotility of the outer hair cell and for the cochlear amplifier. Nature 419, 300-304. doi: 10.1038/nature01059

Liu, X., Bulgakov, O. V., Darrow, K. N., Pawlyk, B., Adamian, M., Liberman, M. C., et al. (2007). Usherin is required for maintenance of retinal photoreceptors and normal development of cochlear hair cells. Proc. Natl. Acad. Sci. U.S.A. 104, 4413-4418. doi: 10.1073/pnas.0610950104

Maison, S. F., Adams, J. C., and Liberman, M. C. (2003). Olivocochlear innervation in the mouse: immunocytochemical maps, crossed versus uncrossed contributions, and transmitter colocalization. J. Comp. Neurol. 455, 406-416. doi: 10.1002/cne. 10490

Maison, S. F., and Liberman, M. C. (2000). Predicting vulnerability to acoustic injury with a noninvasive assay of olivocochlear reflex strength. J. Neurosci. 20, 4701-4707. doi: 10.1523/JNEUROSCI.20-12-04701.2000

Maison, S. F., Liu, X. P., Eatock, R. A., Sibley, D. R., Grandy, D. K., and Liberman, M. C. (2012). Dopaminergic signaling in the cochlea: receptor expression patterns and deletion phenotypes. J. Neurosci. 32, 344-355. doi: 10.1523/ JNEUROSCI.4720-11.2012

Misgeld, T., Burgess, R. W., Lewis, R. M., Cunningham, J. M., Lichtman, J. W., and Sanes, J. R. (2002). Roles of neurotransmitter in synapse formation: development of neuromuscular junctions lacking choline acetyltransferase. Neuron 36, 635-648. doi: 10.1016/s0896-6273(02)01020-6

Nelson, E. G., and Hinojosa, R. (2006). Presbycusis: a human temporal bone study of individuals with downward sloping audiometric patterns of hearing loss and review of the literature. Laryngoscope 116(9 Pt 3 Suppl. 112), 1-12. doi: 10.1097/01.mlg.0000236089.44566.62

Rajan, R., and Johnstone, B. M. (1989). Contralateral cochlear destruction mediates protection from monoaural loud sound exposures through the crossed olivocochlear bundle. Hear. Res. 39, 263-277. doi: 10.1016/0378-5955(89) 90046-4

Sakaguchi, N., Henzl, M. T., Thalmann, I., Thalmann, R., and Schulte, B. A. (1998). Oncomodulin is expressed exclusively by outer hair cells in the organ of Corti. J. Histochem. Cytochem. 46, 29-40. doi: 10.1177/00221554980460 0105

Schwaller, B., Meyer, M., and Schiffmann, S. (2002). 'New' functions for 'old' proteins: the role of the calcium-binding proteins calbindin D$28 \mathrm{k}$, calretinin and parvalbumin, in cerebellar physiology. Studies with knockout mice. Cerebellum 1, 241-258. doi: 10.1080/1473422023208 83551

Simmons, D. D. (2002). Development of the inner ear efferent system across vertebrate species. J. Neurobiol. 53, 228-250. doi: 10.1002/neu.10130

Simmons, D. D., Tong, B., Schrader, A. D., and Hornak, A. J. (2010). Oncomodulin identifies different hair cell types in the mammalian inner ear. J. Comp. Neurol. 518, 3785-3802. doi: 10.1002/cne.22424

Someya, S., Xu, J., Kondo, K., Ding, D., Salvi, R. J., Yamasoba, T., et al. (2009). Age-related hearing loss in C57BL/6J mice is mediated by Bak-dependent mitochondrial apoptosis. Proc. Natl. Acad. Sci. U.S.A. 106, 19432-19437. doi: 10.1073/pnas.0908786106

Spongr, V. P., Flood, D. G., Frisina, R. D., and Salvi, R. J. (1997). Quantitative measures of hair cell loss in CBA and C57BL/6 mice throughout their life spans. J. Acoust. Soc. Am. 101, 3546-3553. doi: 10.1121/1.418315

Sun, X. M., and Kim, D. O. (1999). Adaptation of 2f1-2f2 distortion product otoacoustic emission in young-adult and old CBA and C57 mice. J. Acoust. Soc. Am. 105, 3399-3409. doi: 10.1121/1.424668

Thalmann, R., Henzl, M. T., and Thalmann, I. (1997). Specific proteins of the organ of Corti. Acta Otolaryngol. 117, 265-268. doi: 10.3109/00016489709 117784

Tong, B., Hornak, A. J., Maison, S. F., Ohlemiller, K. K., Liberman, M. C., and Simmons, D. D. (2016). Oncomodulin, an EF-hand Ca2+ buffer, is critical for maintaining cochlear function in mice. J. Neurosci. 36, 1631-1635. doi: 10.1523/JNEUROSCI.3311-15.2016

Wang, J., and Puel, J. L. (2020). Presbycusis: an update on cochlear mechanisms and therapies. J. Clin. Med. 9:22. doi: 10.3390/jcm9010218

Wersinger, E., and Fuchs, P. A. (2011). Modulation of hair cell efferents. Hear. Res. 279, 1-12. doi: 10.1016/j.heares.2010.12.018

Wu, P. Z., Liberman, L. D., Bennett, K., de Gruttola, V., O'Malley, J. T., and Liberman, M. C. (2019). Primary neural degeneration in the human cochlea: 
evidence for hidden hearing loss in the aging ear. Neuroscience 407, 8-20. doi: 10.1016/j.neuroscience.2018.07.053

Wu, P. Z., O'Malley, J. T., de Gruttola, V., and Liberman, M. C. (2020). Agerelated hearing loss is dominated by damage to inner ear sensory cells, not the cellular battery that powers them. J. Neurosci. 40, 6357-6366. doi: 10.1523/ JNEUROSCI.0937-20.2020

Yang, D., Thalmann, I., Thalmann, R., and Simmons, D. D. (2004). Expression of alpha and beta parvalbumin is differentially regulated in the rat organ of corti during development. J. Neurobiol. 58, 479-492. doi: 10.1002/neu.10289

Zheng, X. Y., Henderson, D., McFadden, S. L., and Hu, B. H. (1997). The role of the cochlear efferent system in acquired resistance to noiseinduced hearing loss. Hear. Res. 104, 191-203. doi: 10.1016/s0378-5955(96)0 0187-6

Zhu, X., Vasilyeva, O. N., Kim, S., Jacobson, M., Romney, J., Waterman, M. S., et al. (2007). Auditory efferent feedback system deficits precede age-related hearing loss: contralateral suppression of otoacoustic emissions in mice. J. Comp. Neurol. 503, 593-604. doi: 10.1002/cne.21402
Conflict of Interest: The authors declare that the research was conducted in the absence of any commercial or financial relationships that could be construed as a potential conflict of interest.

Publisher's Note: All claims expressed in this article are solely those of the authors and do not necessarily represent those of their affiliated organizations, or those of the publisher, the editors and the reviewers. Any product that may be evaluated in this article, or claim that may be made by its manufacturer, is not guaranteed or endorsed by the publisher.

Copyright $(2021$ Climer, Hornak, Murtha, Yang, Cox, Simpson, Le and Simmons. This is an open-access article distributed under the terms of the Creative Commons Attribution License (CC BY). The use, distribution or reproduction in other forums is permitted, provided the original author(s) and the copyright owner(s) are credited and that the original publication in this journal is cited, in accordance with accepted academic practice. No use, distribution or reproduction is permitted which does not comply with these terms. 